\title{
Effective Maxwell equations in a geometry with flat rings of arbitrary shape
}

\author{
Agnes Lamacz and Ben Schweizer
}

Preprint 2012-06

April 2012

Fakultät für Mathematik

Technische Universität Dortmund

Vogelpothsweg 87

44227 Dortmund 



\title{
Effective Maxwell equations in a geometry with flat rings of arbitrary shape
}

\author{
Agnes Lamacz ${ }^{1,2}$ and Ben Schweizer ${ }^{1,3}$
}

April 18, 2012

\begin{abstract}
We analyze the time harmonic Maxwell's equations in a complex geometry. The homogenization process is performed in the case that many small, thin conductors are distributed in a subdomain of $\mathbb{R}^{3}$. Each single conductor is, topologically, a split ring resonator, but we allow arbitrary flat shapes. In the limit of large conductivities in the rings and small ring diameters we obtain an effective Maxwell system. Depending on the frequency, the effective system can exhibit a negative effective permeability.
\end{abstract}

MSC: 35Q61, 35B27

key-words: Maxwell equations, homogenization, negative index materials

\section{Introduction}

The description of phenomena regarding the propagation of light receives renewed interest in the last years. This is, on the one hand, due to technical progress that demands for smaller devices and offers new possibilities. On the other hand, some more theoretical investigations are triggered by ideas connected to key-words such as negative index metamaterials, perfect imaging, and cloaking. We are contented to see an increasing interaction between the more technical and the more theoretical approach.

In the field of negative index materials, two very influencial works are the theoretical study of such materials by Veselago in [25], and the approach of Pendry and others to the actual construction of such materials, e.g. [22]. For further material we refer to $[10,21,24]$. The mathematical analysis of such materials is connected to a study of singular limits in Maxwell equations. The possibility of negative effective permittivity was shown mathematically in $[5,6]$. Regarding a negative effective permeability, we refer to an analysis of a two-dimensional model in [13], and to the three-dimensional time-harmonic study in [8].

The contribution at hand continues the investigations of [8], since we derive another homogenization result for the three-dimensional Maxwell scattering problem. In contrast to that work, we analyze here the effective behavior of thin rings. This is of relevance since experiments are usually performed with thin metallic resonators. Within the class of essentially two-dimensional rings, we allow quite general geometries and the methods are

\footnotetext{
${ }^{1}$ TU Dortmund, Fakultät für Mathematik, Vogelpothsweg 87, D-44227 Dortmund, Germany. Support by the DFG, SCHW 639/5-1.

2agnes.lamacz@tu-dortmund.de

${ }^{3}$ ben.schweizer@tu-dortmund.de
} 
not restricted to circular structures that were investigated in [8]. Concerning methods, the generalization helps to identify relevant tools, the new proofs are not taylored to a specific ring geometry.

Problem description. For the sake of clarity, let us give here a problem description in mathematical terms. This article is devoted to the study of the Maxwell equations in a complex geometry. More specifically, we investigate the behavior of the time harmonic Maxwell system in a geometry, where many, highly conducting, small objects are distributed in a periodic fashion. We use the variable $\eta>0$ as an index for a family of coefficients. Investigating small structures then means to study the limiting behavior of solutions as $\eta \rightarrow 0$. The positive number $\eta$ stands for a typical size of the micro-structure. Additionally, the factor $\eta$ enters the values of the permittivity in the conductive material. Finally, as a third singular behavior, the factor $\eta$ enters also in the description of the local behavior of the micro-structure; we impose that the single element of the periodic structure has an aspect ratio which is again connected to some power of $\eta$.

All the complex behavior of the micro-structure can be encoded in one single coefficient, the relative permittivity $\varepsilon_{\eta}$. This paper is concerned with the following problem. Given a sequence of coefficient functions $\varepsilon_{\eta}: \mathbb{R}^{3} \rightarrow \mathbb{C}$ that is related to the slit-ring geometry, let $\left(E^{\eta}, H^{\eta}\right)$ be solutions to the time harmonic Maxwell system

$$
\begin{aligned}
& \operatorname{curl} E^{\eta}=i \omega \mu_{0} H^{\eta}, \\
& \operatorname{curl} H^{\eta}=-i \omega \varepsilon_{\eta} \varepsilon_{0} E^{\eta} .
\end{aligned}
$$

What is, in the limit $\eta \rightarrow 0$, the behavior of the solutions $\left(E^{\eta}, H^{\eta}\right)$ ?

We study (1.1)-(1.2) for a fixed absolute permeability $\mu_{0}>0$, a fixed absolute permittivity $\varepsilon_{0}>0$, and a prescribed frequency $\omega>0$. The relative permittivity is given by a subset $\Sigma_{\eta} \subset \mathbb{R}^{3}$, which defines the complex geometry. Using a constant $\kappa>0$ which is related to the conductivity in the complex structure, we treat the relative permittivity

$$
\varepsilon_{\eta}= \begin{cases}1+i \frac{\kappa}{\eta^{3}} & \text { in } \Sigma_{\eta}, \\ 1 & \text { in } \mathbb{R}^{3} \backslash \Sigma_{\eta}\end{cases}
$$

Further literature. As a tool for the mathematical analysis we will use two-scale convergence as it was developed in $[1,20]$. It allows to separate the macroscopic and the microscopic behavior of oscillatory sequences of functions.

The homogenization of fibres was one of the early applications of the theory, long substructures are important in elasticity problems [2] and in conductivity problems [9]. The relevance for the Maxwell equations has been discovered and explored only later, see [10]. In this application, thin fibres can be used to create materials that behave, effectively, like a medium with negative permittivity.

Regarding a negative permeability, i.e. the investigation of split rings, much less literature is available. Additional to the above-mentioned works [8, 13], we would like to mention [3] for an analysis of dielectrics. For the combined effect of both negative permittivity and negative permeability we refer to [18].

The singular behavior of the substructure can lead to interesting effects in the homogenization process, this was observed also in [16, 23], concerning non-trivial limit equations due to a study of the long-time behavior of solutions see [15]. 
From a more physical point of view, split rings have been analyzed also in $[11,19]$. Regarding possible applications of negative index meta-materials we mention the effect of cloaking, which was investigated e.g. in $[7,14,12,17]$.

Comparison with [8]. The result of the paper at hand is comparable with that of [8]. In both works, an effective Maxwell equation is derived for a singular geometry and for singular parameters. The singularities are chosen in both contributions in such a way that the resulting Maxwell system can have a negative effective permeability.

Some aspects have been studied in more detail in [8]. One concerns the analysis of the scattering problem. In order to conclude such a result from our theorem 1, one has to derive local $L^{2}$-bounds for solution sequences of the scattering problem. This analysis has been performed in [8], and we omit it here. Furthermore, the sign conditions for the effective coefficients have been studied in more detail in [8]. Here, we restrict ourself to a calculation for the formal limiting cell-problem, without deriving mathematically the convergence of solutions.

In two aspects, the paper at hand presents relevant progress in the analysis of the Maxwell system with singular parameters. One concerns the flat ring geometry. We consider here, in accordance with technically manufactured meta-materials, rings that are very thin. While some technical problems have to be resolved to deal with such structures (two-scale convergence with concentration), the cell-problems are actually easier to analyze. A second aspect regards the generality of the geometry. While the analysis of [8] was restricted to precisely circular rings with a very restricted slit geometry, we now generalize to arbitrary ring shapes. Mathematically, this is reflected by the fact that we use a weight function $\theta_{Y}$ in (4.4)-(4.5), and that we cannot use a cell solution at this point. The corresponding generalizations are an important aspect of this work, especially when we think of the modern fish-net designs of meta-materials.

We remark that, in the cell-problem of [8], there was actually missing one contribution. We claimed wrongly in [8] that the jump of the current across the slit in (3.15) necessarily vanishes. We note that we only recover such a condition as a formal limit for $\alpha \rightarrow 0$.

\subsection{Geometry and notation}

In order to complete the problem setting of (1.1)-(1.3), it remains to specify the subset $\Sigma_{\eta}$. This is the main goal of this section. We will follow standard notation of homogenization theory wherever possible. The macroscopic geometry consists, essentially, in a periodic repetition of the microscopic geometry with a periodicity length $\eta$. The microscopic structure is defined as a subset $\Sigma_{Y}^{\eta}$ of the periodicity cell $Y:=Y^{3}:=\left(-\frac{1}{2}, \frac{1}{2}\right)^{3}$. The complex geometry is contained in a scatterer $\Omega \subset \mathbb{R}^{3}$.

Regarding the notation we use the following system for indices. A set with lower index $Y$ is a subset of $Y$. An upper index 1, 2, or 3 indicates the Hausdorff-dimension of the set. For $\eta$-dependent subsets of $Y$ (or other sets that depend on the fast variable $y$ ), we use an upper index $\eta$, for subsets of $\mathbb{R}^{3}$ with slow variable $x$ we use a lower index $\eta$. We use $e_{1}, e_{2}, e_{3}$ for the Euclidian basis vectors of $\mathbb{R}^{3}$.

Microscopic geometry. Our aim is to specify subsets of $Y$ that describe the geometry of the single split ring. The geometry is prescibed by two numbers $\alpha, \beta>0$, a subset $\Sigma \subset Y^{2}:=\left(-\frac{1}{2}, \frac{1}{2}\right)^{2}$, and a straight line $\Gamma \subset \Sigma$. The set $\Sigma$ is a two-dimensional set which is, up to topological transformations, an annulus. 


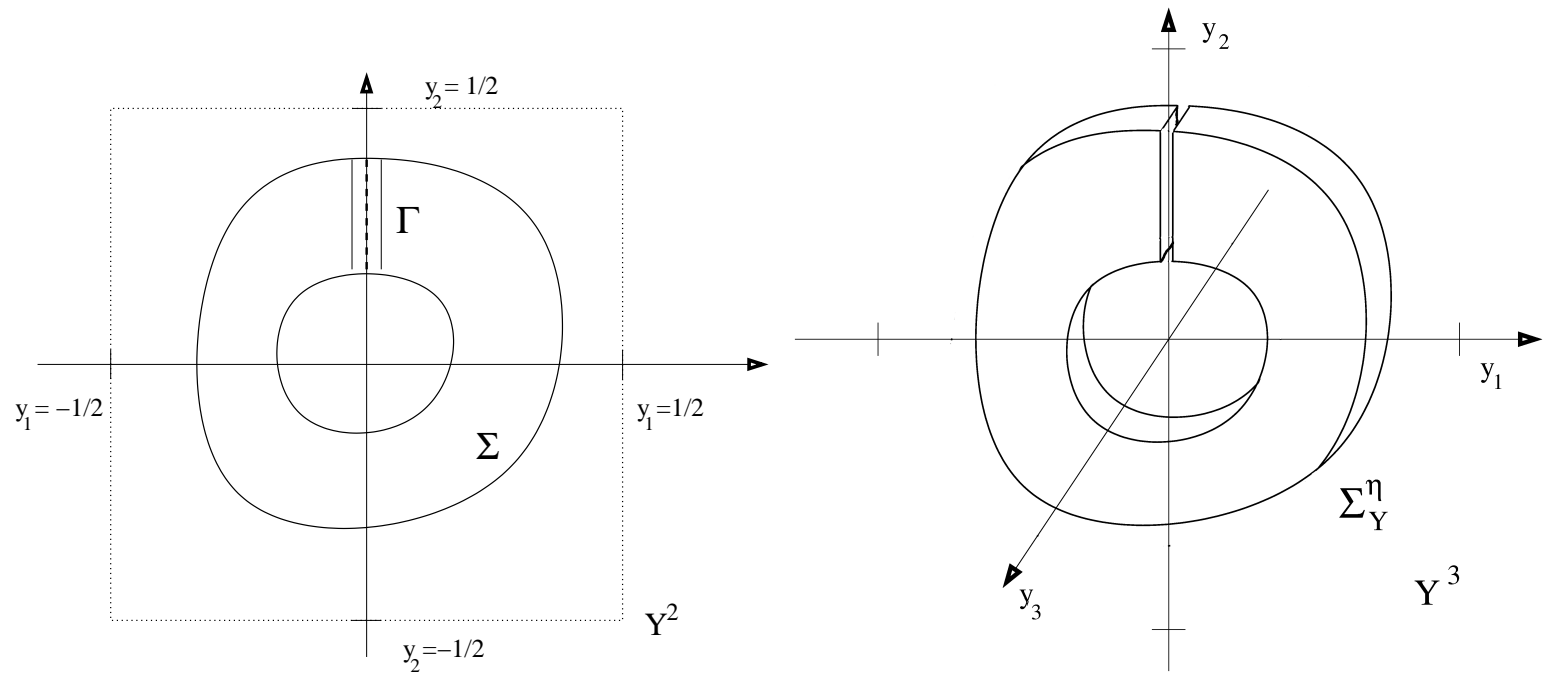

Figure 1: Left: The two-dimensional ring $\Sigma$ with the segment $\Gamma$ indicating the slit position. Right: The three-dimensional ring as a subset of $Y$ with relative thickness $2 \beta \eta$ and slit of thickness $2 \alpha \eta^{3}$.

Further sets are defined as follows. The one-dimensional inner boundary of $\Sigma$ is denoted by $\partial_{i} \Sigma$, the outer boundary is denoted by $\partial_{o} \Sigma$, respectively. By $\Sigma^{\text {inn }}$ we denote the inner connected component of $Y^{2} \backslash \Sigma$. The vector fields $\tau_{\Sigma}$ and $\nu_{\Sigma}$ denote the tangent vector and the normal vector to $\Sigma$ on $\partial \Sigma \subset Y^{2}$.

One-dimensional slit. The slit is constructed starting from the one-dimensional straight line segment $\Gamma \subset \Sigma$. We may write the segment with its tangential vector $\tau_{\Gamma}$ as $\Gamma=$ $\left\{\gamma_{0}+t \tau_{\Gamma}: t \in(0, L(\Gamma))\right\}$ with $\gamma_{0} \in \partial_{i} \Sigma$ and $L(\Gamma)$ denoting the length of the segment $\Gamma$. We assume that $\Gamma$ connects the inner and the outer boundary of $\Sigma$. For ease of notation we assume in the following that inner and outer boundary of $\Sigma$ are straight lines orthogonal to $\tau_{\Gamma}$ in a neighborhood of the end-points of $\Gamma$. Without loss of generality we assume that the tangential vector is $\tau_{\Gamma}=e_{2}$.

Three-dimensional objects. We may regard $\Sigma$ also as a subset of the periodicity cell $Y$. To avoid possible confusion, we denote the set after this identification as $\Sigma_{Y}^{2}:=\Sigma \times\{0\}$. Accordingly, other subsets and vector fields in the plane $Y^{2}$ can be identified with objects in the mid-plane $Y^{2} \times\{0\} \subset Y^{3}$.

Slit construction. A slit of finite size is first constructed in two dimensions as $\Gamma^{2, \eta}:=$ $\Sigma \cap B_{\alpha \eta^{3}}^{2}(\Gamma) \subset Y^{2}$, where $B_{\alpha \eta^{3}}^{2}(\Gamma)$ is the two-dimensional generalized ball with radius $\alpha \eta^{3}$ around $\Gamma$. By our assumption on $\Sigma$, the slit $\Gamma^{2, \eta} \subset \Sigma$ is a rectangle of height $L(\Gamma)$ and width $2 \alpha \eta^{3}$. The two-dimensional ring with slit is constructed as $\Sigma^{\eta}:=\Sigma \backslash \Gamma^{2, \eta}$.

The slit construction is lifted to three dimensions as follows. The three-dimensional closed ring is $\Sigma \times(-\beta \eta, \beta \eta)$, the three-dimensional slit is $\Gamma_{Y}^{\eta}:=\Gamma_{Y}^{3, \eta}:=\Gamma^{2, \eta} \times(-\beta \eta, \beta \eta)$, and the three dimensional ring with narrow slit is defined as

$$
\Sigma_{Y}^{\eta}:=\Sigma_{Y}^{3, \eta}:=\Sigma^{\eta} \times(-\beta \eta, \beta \eta) .
$$

This subset $\Sigma_{Y}^{\eta} \subset Y$ describes the single thin ring with a narrow slit.

Macroscopic geometry. The only macroscopic datum that has to be specified is the open set $\Omega \subset \mathbb{R}^{3}$ that describes the location of the scatterer. For an index $k \in \mathbb{Z}^{3}$ we define 
a shifted small cube as $Y_{k}^{\eta}:=\eta(k+Y)$, and we denote by $\mathcal{K}:=\left\{k \in \mathbb{Z}^{3}: Y_{k}^{\eta} \subset \Omega\right\}$ the set of indices such that the small cube $Y_{k}^{\eta}$ is contained in $\Omega$. Here and in the following, in summations or unions over $k$, the index $k$ takes all values in the index set $\mathcal{K}$. The number of relevant indices has the scaling $|\mathcal{K}|=O\left(\eta^{-3}\right)$. With this notation, the collection of split rings is defined as

$$
\Sigma_{\eta}:=\bigcup_{k} \eta\left(k+\Sigma_{Y}^{\eta}\right)
$$

Other quantities can be defined accordingly, e.g. the collection slits as $\Gamma_{\eta}:=\bigcup_{k} \eta\left(k+\Gamma_{Y}^{\eta}\right)$. We notice that the (three dimensional) volumes of the rings and of the slits have the scaling $\left|\Sigma_{\eta}\right|=O(\eta)$ and $\left|\Gamma_{\eta}\right|=O\left(\eta^{4}\right)$.

Operators. Usually, for a vector $a \in \mathbb{R}^{n}$, the $i$-th component is denoted as $a_{i}$. Due to the many sub- and super-skripts that we have to use, we cannot always indicate components in this simple way. As a substitute, we will indicate the corresponding projection instead. For indices $i, j, k \in\{1,2,3\}, j<k$, we use the projection $\pi^{i}: \mathbb{C}^{3} \rightarrow \mathbb{C}, a \mapsto a_{i}$ to the $i$-th component, and the projection $\pi^{j, k}: \mathbb{C}^{3} \rightarrow \mathbb{C}^{2}, a \mapsto\left(a_{j}, a_{k}\right)$.

On the two sides of the mid-plane $Y^{2} \times\{0\}$, we have the two half-cubes $Y^{+}:=Y^{2} \times$ $\left(0, \frac{1}{2}\right)$ and $Y^{-}:=Y^{2} \times\left(-\frac{1}{2}, 0\right)$. Corresponding to these two subdomains we can evaluate two different trace operators, and we have the two different normal vectors $\nu_{\Sigma}^{ \pm}=\mp e_{3}$ to the surface $\Sigma_{Y}^{2} \subset Y^{3}$. By $t r_{\Sigma^{ \pm}}$we denote the trace on $\Sigma_{Y}^{2}$ from $Y^{ \pm}$. These traces allow to define also the jump over the two-dimensional ring structure $\Sigma_{Y}^{2}$, which we denote with squared brackets. For $u \in L^{2}(Y)$ with $\left.u\right|_{Y^{ \pm}} \in H^{1}\left(Y^{ \pm}\right)$, we set

$$
[u]_{\Sigma}:=\operatorname{tr}_{\Sigma^{+}}(u)-\operatorname{tr}_{\Sigma^{-}}(u) .
$$

The trace of single components of a vector field is abbreviated as $t r_{\Sigma^{ \pm}}^{i}:=\operatorname{tr}_{\Sigma^{ \pm}} \circ \pi^{i}$ and $t r_{\Sigma^{ \pm}}^{j, k}:=t r_{\Sigma^{ \pm}} \circ \pi^{j, k}$. When the trace is identical from both sides we also write simply $t r_{\Sigma}$.

In two space dimensions we use the orthogonal rotation $(\cdot)^{\perp}: \mathbb{C}^{2} \rightarrow \mathbb{C}^{2}$, the mapping with $\left(x_{1}, x_{2}\right)^{\perp}:=\left(-x_{2}, x_{1}\right)$.

\subsection{Main result}

Our main result is the derivation of the effective system of Maxwell equations.

Theorem 1 (Effective equations). For $\alpha, \beta>0$ and sets $\Sigma, \Gamma \subset Y^{2}$ as above, let the local geometry be defined by (1.4). For the bounded open subset $\Omega \subset \mathbb{R}^{3}$, let the split ring geometry be defined by (1.5). With the conductivity parameter $\kappa>0$, let $\varepsilon_{\eta}$ be given by (1.3).

We consider, on a set $Q$ with $\bar{\Omega} \subset Q \subset \mathbb{R}^{3}$, a sequence of solutions $\left(E^{\eta}, H^{\eta}\right)$ to the Maxwell equations (1.1)-(1.2) on $Q$. We assume the boundedness

$$
\int_{Q}\left|H^{\eta}\right|^{2}+\left|E^{\eta}\right|^{2} \leq C
$$

with $C>0$ independent of $\eta$. Let $(E, H)$ be a weak limit of a solution sequence for $\eta \rightarrow 0$. Then $(E, H)$ satisfies in $Q$ the system

$$
\begin{aligned}
\operatorname{curl} E & =i \omega \mu_{0} H, \\
\operatorname{curl}(\hat{M} H) & =-i \omega \varepsilon_{0} \hat{N} E,
\end{aligned}
$$


where the effective matrix fields $\hat{M}$ and $\hat{N}$ are given as

$$
\hat{M}(x)=\left\{\begin{array}{ll}
\mathcal{M} & \text { for } x \in \Omega \\
1 & \text { for } x \in \mathbb{R}^{3} \backslash \Omega
\end{array} \quad \hat{N}(x)= \begin{cases}\mathcal{N} & \text { for } x \in \Omega \\
1 & \text { for } x \in \mathbb{R}^{3} \backslash \Omega\end{cases}\right.
$$

Formulas for the two effective matrices $\mathcal{M}, \mathcal{N} \in \mathbb{C}^{3 \times 3}$ are given in (4.14) and (4.16). They depend on solutions of cell-problems, hence on $\Sigma$ and $\Gamma$, on the parameters $\alpha, \beta, \kappa>0$, and on the frequency $\omega$.

This article is devoted to the proof of Theorem 1. Before we start the proof, we make some remarks on the theorem.

Remark 1. The effective system can be brought into the form of Maxwell's equations, if transformed field variables are used. Assuming that the matrix $\mathcal{M}$ is invertible, we use $\hat{H}:=\hat{M} H$ and $\hat{E}:=E$ as variables and define effective parameters as

$$
\mu_{\mathrm{eff}}(x)=\left\{\begin{array}{ll}
\mathcal{M}^{-1} & \text { for } x \in \Omega \\
1 & \text { for } x \in \mathbb{R}^{3} \backslash \Omega
\end{array} \quad \varepsilon_{\mathrm{eff}}(x)= \begin{cases}\mathcal{N} & \text { for } x \in \Omega \\
1 & \text { for } x \in \mathbb{R}^{3} \backslash \Omega\end{cases}\right.
$$

With this choice, system (1.8)-(1.9) takes the form

$$
\begin{aligned}
& \operatorname{curl} \hat{E}=i \omega \mu_{\mathrm{eff}} \mu_{0} \hat{H}, \\
& \operatorname{curl} \hat{H}=-i \omega \varepsilon_{\mathrm{eff}} \varepsilon_{0} \hat{E} .
\end{aligned}
$$

Outside the scatterer $\Omega$, the transformed fields coincide with the original fields. We can therefore recover the weak limits $(E, H)$ of solutions of system (1.12)-(1.13) from $(E, H)=(\hat{E}, \hat{H})$ on $Q \backslash \Omega$.

Remark 2. With Lemma 4.3, we give a result on the sign of some effective parameters in a limiting case. It shows that the tensor $\mathcal{M}$ can have eigenvalues with negative real part. This is a crucial feature for a negative index meta-material.

Remark 3. On the generality of the theorem and on the scattering problem. The problem of interest in applications is the scattering problem. In this context one is interested in solutions that are defined on $\mathbb{R}^{3}$, and not only on $Q$. Furthermore, the solutions satisfy a boundary condition at infinity, one usually uses the Silver-Müller radiation condition with a prescribed incident field. Theorem 1 provides the effective scattering problem for such a solution sequence, since the weak limit satisfies again the Silver-Müller condition.

One question has to be answered positively in order to conclude as indicated. Does every solution sequence $\left(E^{\eta}, H^{\eta}\right)$ of the scattering problem satisfy the estimate (1.7) on bounded sets? This question has been answered affirmative in [8]. An indirect argument must be used. Under the assumption that the energy is unbounded, we consider a rescaled solution sequence with unit energy. We find a weakly convergent subsequence, which satisfies, by Theorem 1 or its analog, a Maxwell system, and, by the rescaling, it satisfies the scattering problem with vanishing incident field. A uniqueness result for the effective system then provides that the weak limit necessarily vanishes. With a compactness result for the solution sequence, this is in contradiction with the normalized energy. 


\section{The current $J^{\eta}$ and some mathematical tools}

We use two-scale convergence of $[1,20]$. By assumption (1.7), the fields $E^{\eta}$ and $H^{\eta}$ are bounded in $L^{2}(Q)$. By the compactness result with respect to two-scale convergence, we find a subsequence $\eta \rightarrow 0$ and limit functions $E_{0}, H_{0} \in L^{2}\left(Q \times Y, \mathbb{C}^{3}\right)$ such that

$$
\begin{aligned}
& E^{\eta}(x) \rightarrow E_{0}(x, y) \text { weakly in two scales, } \\
& H^{\eta}(x) \rightarrow H_{0}(x, y) \text { weakly in two scales. }
\end{aligned}
$$

Our main task is to identify the equations that are satisfied by $\left(E_{0}, H_{0}\right)$. A useful additional quantity in this analysis is the rescaled dielectric field $J^{\eta}$, which we introduce in the next subsection. The further subsections are devoted to properties of two-scale convergence for thin local structures. This analysis is necessary since the local geometry of our application is singular: the relative (in $Y$ ) thickness of the ring is $O(\eta)$ and the relative slit width is $O\left(\eta^{3}\right)$.

\subsection{Current field and improved bounds}

Besides $E^{\eta}$ and $H^{\eta}$, we consider a third quantity, namely the rescaled dielectric field $J^{\eta}: Q \rightarrow \mathbb{C}^{3}$. We set

$$
J^{\eta}:=\eta \varepsilon_{\eta} E^{\eta}
$$

Lemma 2.1 (Improved estimates). For every compact subdomain $\tilde{Q} \subset \subset Q$ there exists a constant $C$, depending on the $L^{2}(Q)$-bound for $\left(E^{\eta}, H^{\eta}\right)$, but not dependending on $\eta$, such that

$$
\int_{\tilde{Q}} \mu_{0}\left|H^{\eta}\right|^{2}+\left|\varepsilon_{\eta}\right|\left|E^{\eta}\right|^{2} \leq C
$$

Furthermore, there holds the $L^{1}$-estimate

$$
\int_{\tilde{Q}}\left|J^{\eta}\right| \leq C
$$

Proof. Without loss of generality we assume that $\bar{\Omega}$ is contained in $\tilde{Q}$. We use a cutoff function $\psi \in C_{c}^{\infty}(Q, \mathbb{R})$ with $\psi \equiv 1$ on $\tilde{Q}$. To obtain (2.2), we multiply (1.2) with $i \bar{E}^{\eta}(x) \psi(x)$ and integrate over $Q$. We obtain

$$
i \int_{Q} \operatorname{curl} H^{\eta} \bar{E}^{\eta} \psi=\varepsilon_{0} \omega \int_{Q} \varepsilon_{\eta}\left|E^{\eta}\right|^{2} \psi
$$

On the left hand side we integrate by parts and use (1.1),

$$
\begin{aligned}
i \int_{Q} \operatorname{curl} H^{\eta} \bar{E}^{\eta} \psi & =i \int_{Q} H^{\eta} \operatorname{curl} \bar{E}^{\eta} \psi-i \int_{Q} H^{\eta} \bar{E}^{\eta} \wedge \nabla \psi \\
& =\omega \mu_{0} \int_{Q}\left|H^{\eta}\right|^{2} \psi-i \int_{Q} H^{\eta} \bar{E}^{\eta} \wedge \nabla \psi
\end{aligned}
$$

Both integrals are bounded due to the $L^{2}$-bounds for $\left(E^{\eta}, H^{\eta}\right)$. We conclude that both real and imaginary part of the right hand side of (2.4) are bounded. This provides (2.2). 
In order to show (2.3), we first observe that, to leading order in the rings, the coefficient $\varepsilon_{\eta}$ coincides with $i \kappa \eta^{-3}$. By (2.2) and the fact that the volume is $\left|\Sigma_{\eta}\right|=O(\eta)$, we obtain

$$
\begin{aligned}
\int_{\Sigma_{\eta}}\left|J^{\eta}\right| & =\int_{\Sigma_{\eta}}\left|\eta \varepsilon_{\eta} E^{\eta}\right|=\int_{\Sigma_{\eta}}\left|\eta \sqrt{\left|\varepsilon_{\eta}\right|} \sqrt{\left|\varepsilon_{\eta}\right|} E^{\eta}\right| \\
& \leq C\left(\int_{\Sigma_{\eta}} \eta^{2} \kappa \eta^{-3}\right)^{1 / 2}\left\|\sqrt{\left|\varepsilon_{\eta}\right|} E^{\eta}\right\|_{L^{2}\left(\Sigma_{\eta}\right)} \leq C .
\end{aligned}
$$

This shows the $L^{1}(\tilde{Q})$-boundedness of $J^{\eta}$.

\subsection{Two-scale convergence with concentration on lower dimen- sional objects}

In our application, the current $J^{\eta}$ will concentrate, in each periodicity cell, on the twodimensional ring $\Sigma_{Y}^{2}$. We therefore need to use a variant of two-scale convergence that allows for such concentration effects.

In order to motivate the subsequent notion of two-scale convergence with concentration, we start with an example. The example is elementary in the sense that no homogenization is considered. Let $M \subset \mathbb{R}^{2}$ be a bounded two-dimensional set and let $M_{\eta}:=M \times(0, \eta) \subset \mathbb{R}^{3}$ be a thin three-dimensional object, a thickened version of $M$. Let furthermore $U^{\eta}: \mathbb{R}^{3} \rightarrow \mathbb{R}$ be a sequence of functions with the following two properties:

$$
\int_{\mathbb{R}^{3} \backslash M_{\eta}}\left|U^{\eta}\right| \rightarrow 0, \quad \text { and } \quad \eta \int_{M_{\eta}}\left|U^{\eta}\right|^{2} \leq C .
$$

We claim that (2.5) implies the existence of a function $u_{0} \in L^{2}(M)$ such that, along a subsequence $\eta \rightarrow 0$,

$$
\int_{\mathbb{R}^{3}} U^{\eta}(x) \varphi(x) d x \rightarrow \int_{M} \varphi\left(x_{1}, x_{2}, 0\right) u_{0}\left(x_{1}, x_{2}\right) d x_{1} d x_{2}
$$

for every test function $\varphi \in C_{c}\left(\mathbb{R}^{3}\right)$.

The verification of (2.6) is not difficult. The Cauchy-Schwarz inequality provides an $L^{1}\left(\mathbb{R}^{3}\right)$ bound for the sequence $U^{\eta}$, the weak-* compactness of measures implies the existence of a limit measure $\mu \in \mathcal{M}\left(\mathbb{R}^{3}\right)$. We have, along a subsequence $\eta \rightarrow 0$,

$$
\int_{\mathbb{R}^{3}}\left|U^{\eta}\right| \leq \int_{\mathbb{R}^{3} \backslash M_{\eta}}\left|U^{\eta}\right|+\left(\int_{M_{\eta}}\left|U^{\eta}\right|^{2}\right)^{1 / 2}\left|M_{\eta}\right|^{1 / 2} \leq C, \quad \int_{\mathbb{R}^{3}} U^{\eta}(x) \varphi(x) d x \rightarrow \int_{\mathbb{R}^{3}} \varphi d \mu .
$$

It remains to verify the existence of an $L^{2}(M)$-density $u_{0}$ for the measure $\mu$. We use rescaled averages of $U^{\eta}$ as new functions $u^{\eta}: M \rightarrow \mathbb{R}$, and calculate with Jensen's inequality

$$
u^{\eta}\left(x_{1}, x_{2}\right):=\int_{0}^{\eta} U^{\eta}\left(x_{1}, x_{2}, x_{3}\right) d x_{3}, \quad \int_{M}\left|u^{\eta}\right|^{2} \leq \int_{M} \eta \int_{0}^{\eta}\left|U^{\eta}\right|^{2} \leq C .
$$

This $L^{2}(M)$-bound for $u^{\eta}$ yields, possibly for a further subsequence, the convergence $u^{\eta} \rightarrow u_{0}$ weakly in $L^{2}(M)$. It is easily verified that $u_{0}$ provides a density function for $\mu$. 
Summarizing, we see that the first property of (2.5) implies a concentration on the lower-dimensional set $M$. On the other hand, the second property of (2.5) implies that the values of the sequence $U^{\eta}$ are of order $\eta^{-1}$, and that they are well-distributed over the set $M$.

The next result transfers the above idea to homogenization problems with thin substructures. We will not give an independent proof of Proposition 2.1, but conclude the lemma from a more general result on two-scale convergence with respect to measures, see [4].

We will apply the lemma with $m=2$ and $n=3$, with thin object $\Sigma_{Y}^{\eta}=\Sigma \times(-\beta \eta, \beta \eta)$ of volume $V^{\eta}=\mathcal{L}^{n}\left(\Sigma^{\eta}\right)=O(\eta)$. We write here $\chi_{A}$ for the characteristic function, taking the value 1 in $A$ and 0 outside $A$.

Proposition 2.1 (Two-scale convergence with concentration). For dimensions $1 \leq m \leq$ $n$ and $d \in \mathbb{N}$ let $\Sigma \subset Y=\left(-\frac{1}{2}, \frac{1}{2}\right)^{n}$ be a subset of Hausdorff-dimension $m$. Let $\Sigma^{\eta} \subset Y$ be a sequence of $n$-dimensional objects approximating $\Sigma$ in the sense of

$$
V^{\eta}:=\mathcal{L}^{n}\left(\Sigma^{\eta}\right)>0, \quad \frac{1}{V^{\eta}} \chi_{\Sigma^{\eta}} \stackrel{*}{\rightarrow} \mathcal{H}_{\Sigma}^{m} \text { in } \mathcal{M}(Y) .
$$

We denote by $\Sigma_{\eta}:=\bigcup_{j \in \mathbb{Z}^{n}} \eta\left(j+\Sigma^{\eta}\right)$ the periodic distribution of scaled copies of $\Sigma^{\eta}$ over $\mathbb{R}^{n}$. We consider a sequence of functions $J^{\eta}: \mathbb{R}^{n} \rightarrow \mathbb{R}^{d}$ with supports contained in a fixed bounded set $Q \subset \mathbb{R}^{n}$ satisfying

$$
\begin{array}{ll}
\text { (concentration) } & \int_{Q \backslash \Sigma_{\eta}}\left|J^{\eta}\right| \rightarrow 0 \text { for } \eta \rightarrow 0, \\
\text { (equi-distribution) } & V^{\eta} \int_{Q \cap \Sigma_{\eta}}\left|J^{\eta}\right|^{2} d x \leq C .
\end{array}
$$

Under these conditions, there exists a two-scale limit density $j_{0} \in L_{d x \otimes d \mathcal{H}^{m}}^{2}\left(Q \times \Sigma ; \mathbb{R}^{d}\right)$, such that for every test-function $\varphi \in C_{c}^{\infty}\left(Q ; C_{\text {per }}^{\infty}\left(Y ; \mathbb{R}^{d}\right)\right)$ the following holds

$$
\lim _{\eta \rightarrow 0} \int_{Q} J^{\eta}(x) \cdot \varphi\left(x, \frac{x}{\eta}\right) d x=\int_{Q} \int_{\Sigma} j_{0}(x, y) \cdot \varphi(x, y) d \mathcal{H}^{m}(y) d x .
$$

Proof. We note that conditions (2.8)-(2.9) ensure that $J^{\eta}$ is uniformly bounded in $L^{1}(Q)$. This is shown with the Cauchy-Schwarz inequality just in our preceeding example.

We will now derive Proposition 2.1 from Proposition 2.3 of [4]. To put our application into the frame-work of [4], we define the following functions and measures on the unit cube $Y$ and on $B:=Q$,

$$
\mu_{\eta}:=\frac{1}{V^{\eta}} \chi_{\Sigma^{\eta}}, \quad \mu_{\eta}\left(\frac{x}{\eta}\right):=\frac{1}{V^{\eta}} \chi_{\Sigma_{\eta}}, \quad \mu:=\mathcal{H}_{\Sigma}^{m}, \quad v_{\eta}(x):=V^{\eta} J^{\eta} \chi_{\Sigma_{\eta}} .
$$

With this choice, the sequence $v_{\eta}$ satisfies

$$
\int_{Q}\left|v_{\eta}\right|^{2} d \mu_{\eta}\left(\frac{x}{\eta}\right)=\frac{1}{V^{\eta}} \int_{Q \cap \Sigma_{\eta}}\left(V^{\eta}\right)^{2}\left|J^{\eta}\right|^{2} d x \leq C
$$

by (2.9), hence the assumption of Proposition 2.3 of [4] is satisfied. That proposition provides the existence of a two-scale limit in the sense of a measure, the existence of a 
density $v_{0} \equiv j_{0} \in L_{d x \otimes d \mathcal{H}^{m}}^{2}\left(Q \times \Sigma ; \mathbb{R}^{d}\right)$ and, in particular, the convergence property

$$
\begin{aligned}
\lim _{\eta \rightarrow 0} \int_{Q \cap \Sigma_{\eta}} J^{\eta}(x) \cdot \varphi\left(x, \frac{x}{\eta}\right) d x=\lim _{\eta \rightarrow 0} \int_{Q} v_{\eta}(x) \cdot \varphi\left(x, \frac{x}{\eta}\right) d \mu_{\eta}\left(\frac{x}{\eta}\right) \\
\quad=\int_{Q} \int_{Y} v_{0}(x, y) \cdot \varphi(x, y) d \mu(y) d x=\int_{Q} \int_{\Sigma} j_{0}(x, y) \cdot \varphi(x, y) d \mathcal{H}^{m}(y) d x .
\end{aligned}
$$

This shows (2.10).

With the subsequent lemma we apply Proposition 2.1 to our setting and state the consequence for the sequence of currents $J^{\eta}=\eta \varepsilon_{\eta} E^{\eta}$ in the Maxwell problem. The result is the existence of a limiting current density $j_{0} \in L^{2}\left(\Omega \times \Sigma ; \mathbb{C}^{3}\right)$.

Lemma 2.2 (Two-scale limit of the current $J^{\eta}$ ). Let $\Sigma$ be the two-dimensional ring and let $Y$ be the three-dimensional unit cell. Let the situation be that of Theorem 1, $\left(E^{\eta}, H^{\eta}\right)$ a sequence of solutions to the Maxwell equations and $J^{\eta}$ the corresponding currents. Then there exists a density $j_{0} \in L^{2}\left(\Omega \times \Sigma ; \mathbb{C}^{3}\right)$ such that

$$
\lim _{\eta \rightarrow 0} \int_{Q} J^{\eta}(x) \cdot \varphi\left(x, \frac{x}{\eta}\right) d x=\int_{\Omega} \int_{\Sigma} \varphi\left(x, y_{1}, y_{2}, 0\right) \cdot j_{0}\left(x, y_{1}, y_{2}\right) d\left(y_{1}, y_{2}\right) d x
$$

for every test function $\varphi \in C_{c}^{\infty}\left(Q ; C_{\mathrm{per}}^{\infty}\left(Y ; \mathbb{R}^{3}\right)\right)$.

Proof. The construction of the set $\Sigma_{\eta}$ is such that the geometrical assumptions of Proposition 2.1 are satisfied. By definition of the current $J^{\eta}$ and by the choice of the permittivity $\varepsilon_{\eta}$, there holds $J^{\eta}=\eta \varepsilon_{\eta} E^{\eta}=\eta E^{\eta}+i \frac{\kappa}{\eta^{2}} E^{\eta} \chi_{\Sigma_{\eta} \cap \Omega}$. This provides immediately

$$
\int_{Q \backslash \Sigma_{\eta}}\left|J^{\eta}\right| \leq \int_{Q \backslash \Sigma_{\eta}} \eta\left|E^{\eta}\right| \rightarrow 0
$$

by the uniform $L^{2}(Q)$-bound for $E^{\eta}$. In particular, assumption (2.8) of Proposition 2.1 is satisfied. Regarding the $L^{2}$-assumption (2.9) we calculate, using $V^{\eta}=O(\eta)$ and Lemma 2.1 in the last inequality,

$$
V^{\eta} \int_{Q \cap \Sigma_{\eta}}\left|J^{\eta}\right|^{2} \leq C \eta \int_{Q \cap \Sigma_{\eta}} \eta^{-4}\left|E^{\eta}\right|^{2} \leq C \int_{Q \cap \Sigma_{\eta}}\left|\varepsilon_{\eta}\right|\left|E^{\eta}\right|^{2} \leq C .
$$

This shows that all assumptions of Proposition 2.1 are satisfied. The assertion of that proposition provides the limit density $j_{0} \in L^{2}\left(\Omega \times \Sigma ; \mathbb{C}^{3}\right)$. Relation (2.10) reduces to (2.11).

\subsection{Trace lemma for two-scale limits}

Lemma 2.3 (Trace lemma). Let $N \subset \subset\left(-\frac{1}{2}, \frac{1}{2}\right)^{2}$ a surface which we identify with $N^{0}=$ $N \times\{0\} \subset Y$. For $\zeta \in\left(-\frac{1}{2}, \frac{1}{2}\right)$ we denote the shifted surface by $N^{\zeta}:=N \times\{\zeta\} \subset Y$. Let $N_{\eta}^{\zeta}$ be the union of scaled surfaces, $N_{\eta}^{\zeta}:=\bigcup_{k} \eta\left(k+N^{\zeta}\right)$. The normal vector to all surfaces is $\nu=e_{3}$. Below, we always consider smooth test-function $\varphi_{0}: B \times Y \rightarrow \mathbb{R}$, supported on $N \times\left(-\frac{1}{2}, \frac{1}{2}\right)$.

In a domain $B \subset \mathbb{R}^{3}$, let $H^{\eta}$ be a bounded sequence in $L^{2}\left(B, \mathbb{R}^{3}\right)$ with vanishing divergence, $\nabla \cdot H^{\eta}=0$. In particular, all traces below are well-defined. Then the following holds: 
1.) The traces of $H^{\eta} \cdot e_{3}$ are essentially independent of the plane-positition $\zeta$. More precisely, for every $z \in \mathbb{R}$, there holds

$$
\left|\eta \int_{N_{\eta}^{z \eta}} H^{\eta}(x) \cdot \nu \varphi_{0}(x, x / \eta) d \mathcal{H}^{2}(x)-\eta \int_{N_{\eta}^{0}} H^{\eta}(x) \cdot \nu \varphi_{0}(x, x / \eta) d \mathcal{H}^{2}(x)\right| \rightarrow 0 .
$$

2.) The traces can be evaluated from the two-scale limit. If the sequence $H^{\eta}$ converges weakly in the sense of two scales in $L^{2}(B \times Y)$ to a limit function $H_{0}=H_{0}(x, y)$, then for every $z \in \mathbb{R}$, there holds

$$
\eta \int_{N_{\eta}^{z \eta}} H^{\eta}(x) \cdot \nu \varphi_{0}(x, x / \eta) d \mathcal{H}^{2}(x) \rightarrow \int_{B} \int_{N^{0}} H_{0}(x, y) \cdot \nu(y) \varphi_{0}(x, y) d \mathcal{H}^{2}(y) d x
$$

3.) Volume averages. Using the volumes $V=N \times(-\beta \eta, \beta \eta) \subset \subset Y$ and $V_{\eta}:=$ $\bigcup_{k} \eta(k+V)$, there holds the following convergence for averages,

$$
\frac{1}{\eta} \int_{V_{\eta}} H^{\eta}(x) \cdot \nu \varphi_{0}(x, x / \eta) d x \rightarrow 2 \beta \int_{B} \int_{N^{0}} H_{0}(x, y) \cdot \nu(y) \varphi_{0}(x, y) d \mathcal{H}^{2}(y) d x .
$$

Proof. By density, it is sufficient to consider only test-functions $\varphi_{0}(x, y)=\psi(x) \varphi(y)$ with $\varphi \in C_{c}^{\infty}(Y)$.

1.) We use the definition of the trace. Setting $\varphi_{+}^{z \eta}(y)=\varphi(y)$ for $y_{3}>z \eta$ and $\varphi_{+}^{z \eta}(y)=0$ for $y_{3} \leq z \eta$, we can write, intending only the regular part of the derivatives in bulk integrals,

$$
\begin{gathered}
\eta \int_{N_{\eta}^{z \eta}} H^{\eta}(x) \cdot \nu \varphi_{0}(x, x / \eta) d \mathcal{H}^{2}(x)=-\eta \int_{Q} \nabla \cdot\left(H^{\eta}(x) \psi(x) \varphi_{+}^{z \eta}(x / \eta)\right) \\
=-\int_{Q} H^{\eta}(x) \cdot \nabla_{y} \varphi_{+}^{z \eta}(x / \eta) \psi(x) d x+o(1) .
\end{gathered}
$$

The difference $\nabla_{y} \varphi_{+}^{z \eta}-\nabla_{y} \varphi_{+}^{0}$ vanishes everywhere except for a set of vanishing measure in $Y$. The $L^{2}$-bound for $H^{\eta}$ implies the claim of (2.12).

2.) The limit (2.13) is again a consequence of the definition of the surface integral. In view of (2.12) it suffices to calculate for $z=0$. We evaluate the surface integrals with the help of volume integrals over one half of the cubes, setting $Y^{+}:=\left\{x \in Y \mid y_{3}>0\right\}$ and $Y_{\eta}^{+}:=\bigcup_{k} \eta\left(k+Y^{+}\right)$. This allows to calculate

$$
\begin{aligned}
& \eta \int_{N_{\eta}^{0}} H^{\eta} \cdot \nu \varphi_{0}(x, x / \eta) d \mathcal{H}^{2}(x)=-\int_{Y_{\eta}^{+}} H^{\eta} \cdot \nabla_{y} \varphi(x / \eta) \psi(x) d x+o(1) \\
& \rightarrow-\int_{B} \int_{Y^{+}} H_{0}(x, y) \cdot \nabla_{y} \varphi(y) \psi(x) d y d x=\int_{B} \int_{N^{0}} H_{0}(x, y) \cdot \nu(y) \varphi_{0}(x, y) d \mathcal{H}^{2}(y) d x
\end{aligned}
$$

for $\eta \rightarrow 0$. This provides relation (2.13).

3.) The volume averages are calculated with Fubini's theorem as

$$
\begin{aligned}
\frac{1}{\eta} \int_{V_{\eta}} & H^{\eta} \cdot \nu \varphi_{0}(x, x / \eta) d x=\int_{-\beta \eta}^{\beta \eta} \int_{N_{\eta}^{\zeta}} H^{\eta} \cdot \nu \varphi_{0}(x, x / \eta) d \mathcal{H}^{2}(x) d \zeta \\
\quad & \int_{-\beta}^{\beta}\left(\eta \int_{N_{\eta}^{z \eta}} H^{\eta} \cdot \nu \varphi_{0}(x, x / \eta) d \mathcal{H}^{2}(x)\right) d z \\
& \rightarrow 2 \beta \int_{B} \int_{N^{0}} H_{0}(x, y) \cdot \nu(y) \varphi_{0}(x, y) d \mathcal{H}^{2}(y) d x,
\end{aligned}
$$

where the convergence for $\eta \rightarrow 0$ follows from (2.12) and (2.13). 


\section{The cell problems}

With the tools of section 2 we can now start with the proof of Theorem 1 . In the following, we will always consider the situation of that theorem, with $\left(E^{\eta}, H^{\eta}\right)$ a sequence of solutions to the Maxwell equations and $J^{\eta}$ the corresponding currents. Upon choosing a subsequence, we may assume the two-scale convergences to limit functions $\left(E_{0}, H_{0}\right)$ and to a limit current density $j_{0}$ as follows,

$$
\begin{aligned}
& \lim _{\eta \rightarrow 0} \int_{Q} E^{\eta}(x) \varphi\left(x, \frac{x}{\eta}\right) d x=\int_{Q} \int_{Y} E_{0}(x, y) \varphi(x, y) d y d x \\
& \lim _{\eta \rightarrow 0} \int_{Q} H^{\eta}(x) \varphi\left(x, \frac{x}{\eta}\right) d x=\int_{Q} \int_{Y} H_{0}(x, y) \varphi(x, y) d y d x \\
& \lim _{\eta \rightarrow 0} \int_{Q} J^{\eta}(x) \varphi\left(x, \frac{x}{\eta}\right) d x=\int_{Q} \int_{\Sigma} \varphi\left(x, y_{1}, y_{2}, 0\right) j_{0}\left(x, y_{1}, y_{2}\right) d\left(y_{1}, y_{2}\right) d x
\end{aligned}
$$

for every test function $\varphi \in C_{c}^{\infty}\left(Q ; C_{\text {per }}^{\infty}\left(Y ; \mathbb{R}^{3}\right)\right)$. This section is devoted to the derivation of limit equations for $\left(E_{0}, H_{0}\right)$ and $j_{0}$ in subsection 3.1 and to the analysis of those limit equations in subsection 3.2 .

\subsection{Derivation of the cell problems}

In this subsection we derive the cell problems for the two scale limit $\left(E_{0}, H_{0}, j_{0}\right)$. We note that outside the scatterer $\Omega$, i.e. for $x \in Q \backslash \Omega$, the sequences $E^{\eta}$ and $H^{\eta}$ converge strongly and the two-scale limits are independent of $y$.

\section{Cell-problem for $E_{0}$}

Lemma 3.1 (Cell-problem for $\left.E_{0}\right)$. Let $\left(E^{\eta}, H^{\eta}\right)$ be a sequence of solutions of the Maxwell equations as described in Theorem 1, and let $E_{0}(x, y)$ be a two-scale limit of $E^{\eta}$. Then, for almost every $x \in \Omega$, the function $E_{0}=E_{0}(x,$.$) satisfies, on the torus Y$,

$$
\begin{aligned}
\operatorname{curl}_{y} E_{0} & =0 \text { in } Y, \\
\operatorname{div}_{y} E_{0} & =0 \text { in } Y \backslash \bar{\Sigma}_{Y}^{2}, \\
\operatorname{tr}_{\Sigma}^{1,2} E_{0} & =0 \text { in } \Sigma_{Y}^{2} .
\end{aligned}
$$

Proof. The derivation of (3.4)-(3.5) is straight-forward. According to the spirit of twoscale convergence, we consider arbitrary functions $\psi \in C_{c}^{\infty}(\Omega ; \mathbb{R})$ and $\theta \in C_{\text {per }}^{\infty}\left(Y ; \mathbb{R}^{3}\right)$ and consider $\eta \psi(x) \theta(x / \eta)$ as test-functions in (1.1)-(1.2). The function $\theta$ is chosen with support in $Y \backslash \bar{\Sigma}_{Y}^{2}$ in order to derive (3.5).

It remains to verify the trace statement (3.6). Before we perform the calculation, we note that the trace of (the tangential components of) $E_{0}$ on the two-dimensional set $\Sigma_{Y}^{2}$ is well-defined in the distributional sense since $E_{0}$ is in $L^{2}(Y)$ and curl-free by (3.4). In particular, the trace is independent of the side from which it is evaluated.

Step 1: Construction of special test-functions. We must derive (3.6) from the fact that $E^{\eta}$ has very small values in $\Sigma_{\eta}$. This smallness is expressed with the improved bound (2.2), since $\varepsilon_{\eta}$ has large values in $\Sigma_{\eta}$.

In order to derive a conclusion from (2.2), we construct a special sequence of test functions with large gradients. We start the construction from an arbitrary function $\theta \in C_{c}^{\infty}\left(Y^{2}, \mathbb{R}^{3}\right)$ with support in $\Sigma$. We extend $\theta$ with a function $\varphi$ to the cube $Y^{3}$ with 
support in the half-cube $Y^{2} \times\left[0, \frac{1}{2}\right)$. More precisely, we choose $\varphi(y)=\theta\left(y_{1}, y_{2}\right) \phi\left(y_{3}\right)$, where $\phi:\left(-\frac{1}{2}, \frac{1}{2}\right) \rightarrow \mathbb{R}$ is the trivial (discontinuous) extension of a function $\tilde{\phi} \in C_{c}^{\infty}\left(\left[0, \frac{1}{2}\right), \mathbb{R}\right)$ with $\tilde{\phi} \equiv 1$ in a neighborhood of $y_{3}=0$.

Additionally, we use the curl of $\varphi$, setting

$$
\vartheta:= \begin{cases}\operatorname{curl} \varphi & \text { in } Y^{+} \\ 0 & \text { in } Y^{-}\end{cases}
$$

With this choice, $\vartheta$ is the regular part (with respect to the Lebesgue measure) of curl $\varphi$.

We can now define a continuous function $\varphi^{\eta}: Y \rightarrow \mathbb{R}^{3}$ with compact support in $Y$. For $\eta>0$ sufficiently small we set

$$
\varphi^{\eta}\left(y_{1}, y_{2}, y_{3}\right):= \begin{cases}\varphi\left(y_{1}, y_{2}, y_{3}-\beta \eta\right) & \text { for } y_{3} \geq \beta \eta \\ \theta\left(y_{1}, y_{2}\right) y_{3}(\beta \eta)^{-1} & \text { for } y_{3} \in(0, \beta \eta) \\ 0 & \text { for } y_{3} \leq 0\end{cases}
$$

The gradient of this function has the scaling $\left\|\nabla \varphi^{\eta}\right\|_{L^{\infty}(Y)}=O\left(\eta^{-1}\right)$.

Step 2: Evaluation of limit traces. In the following, we use an arbitrary function $\psi \in C_{c}^{\infty}(\Omega ; \mathbb{R})$ to localize the result in $x$. As indicated before, the tangential traces of $E_{0}$ on $\Sigma$ vanish due to the smallness of the electric field in the metallic structure. We exploit the corresponding estimate (2.2) by calculating, with the Cauchy-Schwarz inequality,

$$
\left|\int_{\Sigma_{\eta}} E^{\eta}(x) \cdot \operatorname{curl}_{y} \varphi^{\eta}(x / \eta) \psi(x) d x\right| \leq C\left|\Sigma_{\eta}\right|^{1 / 2} \frac{1}{\eta}\left(\int_{\Sigma_{\eta}}\left|E^{\eta}\right|^{2}\right)^{1 / 2} \leq C \eta^{1 / 2} \eta^{-1} \eta^{3 / 2} \rightarrow 0
$$

for $\eta \rightarrow 0$, where we used $\left|\Sigma_{\eta}\right| \leq C \eta$ and $\left|\varepsilon_{\eta}\right|=O\left(\eta^{-3}\right)$ in $\Sigma_{\eta}$. In order to draw further conclusions, we extend the integral on the left hand side to all of $\Omega$ and integrate by parts, obtaining

$$
\int_{\Omega \backslash \Sigma_{\eta}} E^{\eta}(x) \cdot \operatorname{curl}_{y} \varphi^{\eta}(x / \eta) \psi(x) d x=\eta \int_{\Omega} \operatorname{curl}_{x} E^{\eta}(x) \cdot \varphi^{\eta}(x / \eta) \psi(x) d x+o(1) \rightarrow 0
$$

for $\eta \rightarrow 0$, where we used, concerning the limit of the integral on the right hand side, that $\operatorname{curl}_{x} E^{\eta}=i \omega \mu_{0} H^{\eta}$ is bounded in $L^{2}(Q)$.

It remains to evaluate the left hand side of (3.7) with two-scale convergence. Inserting the curl function $\theta$ we obtain, for $\eta \rightarrow 0$,

$$
\begin{aligned}
0 \leftarrow & \int_{\Omega \backslash \Sigma_{\eta}} E^{\eta}(x) \cdot \operatorname{curl}_{y} \varphi^{\eta}(x / \eta) \psi(x) d x=\int_{\Omega \backslash \Sigma_{\eta}} E^{\eta}(x) \cdot \vartheta(x / \eta) \psi(x) d x \\
& +\int_{\Omega \backslash \Sigma_{\eta}} E^{\eta}(x) \cdot\left(\operatorname{curl}_{y} \varphi^{\eta}-\vartheta\right)(x / \eta) \psi(x) d x \rightarrow \int_{\Omega} \int_{Y} E_{0}(x, y) \vartheta(y) d y \psi(x) d x .
\end{aligned}
$$

In the last limit we exploited that $\vartheta$ is obtained from $\operatorname{curl}_{y} \varphi^{\eta}$ by a shift of size $\eta$, hence the integral containing $\operatorname{curl}_{y} \varphi^{\eta}-\vartheta$ tends to zero. For the other integral, the definition of two-scale convergence is used to evaluate the limit. With another integration by parts we conclude, for almost every $x \in \Omega$, using $\operatorname{curl}_{y} E_{0}(x, y)=0$ in $Y$,

$$
\begin{aligned}
0 & =\int_{Y} E_{0}(x, y) \cdot \vartheta(y) d y=\int_{Y^{+}} E_{0}(x, y) \cdot \operatorname{curl}_{y} \varphi(y) d y \\
& =\int_{\Sigma}\left(-e_{3} \wedge \theta\left(y_{1}, y_{2}\right)\right) \cdot \operatorname{tr}_{\Sigma^{+}}^{1,2} E_{0}(x, y) d y_{1} d y_{2} \\
& =\int_{\Omega} \int_{\Sigma} \operatorname{tr}_{\Sigma}^{1,2} E_{0}(x, y) \cdot\left(\begin{array}{c}
\pi^{2} \theta \\
-\pi^{1} \theta
\end{array}\right)\left(y_{1}, y_{2}\right) d y_{1} d y_{2} .
\end{aligned}
$$


Since the two first components $\pi^{1} \theta$ and $\pi^{2} \theta$ of $\theta$ were arbitrary, equation (3.6) is shown.

Representation of the electric field $E_{0}(x, y)$. We claim that the solution space for system $(3.4)-(3.6)$ is three-dimensional. Even more, every solution $E_{0}(x, \cdot)$ of that system can be reconstructed from the three components of the average

$$
E(x)=f_{Y} E_{0}(x, y) d y
$$

Indeed, equation (3.4) implies that $E_{0}$ is a gradient on $Y$, we write $E_{0}(x, \cdot)=E(x)+$ $\nabla_{y} \phi(x, \cdot)$, where $\phi$ is a scalar periodic potential in $W_{\text {per }}^{1,2}(Y, \mathbb{C})$. Equation (3.5) implies that $\phi$ is harmonic in $Y \backslash \bar{\Sigma}_{Y}^{2}$. Equation (3.6) implies that $\pi^{1,2}(E(x)+\nabla \phi)$ vanishes on $\Sigma_{Y}^{2}$. With these two relations, the potential $\phi$ is, up to additive constants, uniquely determined by the vector $E(x) \in \mathbb{C}^{3}$. In particular, by linearity of the equations, the three components $E_{1}(x), \ldots E_{3}(x)$ determine $E_{0}(x, \cdot)$ and we conclude that the microscopic field can be written as

$$
E_{0}(x, y)=\sum_{k=1}^{3} E_{k}(x) E^{k}(y) .
$$

The shape functions $E^{k}(y):=e_{k}+\nabla \phi^{k}(y)$ are real valued and can be obtained by solving

$$
\begin{aligned}
\Delta \phi^{k} & =0 \quad \text { on } Y \backslash \bar{\Sigma}_{Y}^{2}, \\
\operatorname{tr}_{\Sigma} \phi^{k}\left(y_{1}, y_{2}, 0\right) & =-\left(y_{1}, y_{2}, 0\right) \cdot e_{k} \quad \text { on } \Sigma_{Y}^{2} .
\end{aligned}
$$

\section{Cell-problem for $\left(H_{0}, j_{0}\right)$}

In the next lemma we state and derive the cell-problem for the magnetic field and the current density. This cell problem is much more interesting than the cell problem for the electric field. The reason lies in the geometry of the rings: we do obtain $\operatorname{curl}_{y} H_{0}(x,)=$. in $Y \backslash \bar{\Sigma}_{Y}^{2}$, but we cannot conclude that $H_{0}$ is a gradient, since the set $Y \backslash \bar{\Sigma}_{Y}^{2}$ is not simply connected. Loosely speaking, it is the non-trivial vector field, which is curl-free but not a gradient, that introduces the extra dimension in the magnetic cell problem. Later on, we will see that the cell problem of Lemma 3.2 has a four-dimensional solution space.

Lemma 3.2 (Cell-problem for the pair $\left.\left(H_{0}, j_{0}\right)\right)$. Let $\left(E^{\eta}, H^{\eta}\right)$ be a sequence of solutions as in Theorem 1. Let $H_{0}(x, y)$ be a two-scale limit of $H^{\eta}$ and let $j_{0} \in L^{2}\left(\Omega \times \Sigma ; \mathbb{C}^{3}\right)$ be a limit density for $J^{\eta}$ as in (3.2)-(3.3). Then, for almost every $x \in \Omega$, the limit current is two-dimensional, satisfying $\pi^{3} j_{0}=0$. With an identification and upon extending $j_{0}$ by zero to $Y^{2}$ we may also write $j_{0} \in L^{2}\left(\Omega \times Y^{2} ; \mathbb{C}^{2}\right)$. The functions $H_{0}=H_{0}(x, \cdot)$ and $j_{0}=j_{0}(x, \cdot)$ satisfy the following cell problem (3.10)-(3.15).

$$
\begin{aligned}
\operatorname{div}_{y} H_{0} & =0 & & \text { in } Y \\
\operatorname{curl}_{y} H_{0} & =0 & & \text { in } Y \backslash \bar{\Sigma}_{Y}^{2} \\
\nabla_{\left(y_{1}, y_{2}\right)} \cdot j_{0} & =0 & & \text { on } Y^{2} \\
{\left[\left(\pi^{1,2} H_{0}\right)^{\perp}\right]_{\Sigma} } & =-i \omega \varepsilon_{0} j_{0} & & \text { on } \Sigma \\
\nabla_{\left(y_{1}, y_{2}\right)}^{\perp} \cdot j_{0} & =-2 \beta \kappa \omega \mu_{0} t r_{\Sigma}^{3} H_{0} & & \text { on } \Sigma \backslash \Gamma \\
{\left[\pi^{2} j_{0}\right]_{\Gamma} } & =2 \alpha i \kappa \partial_{y_{2}}\left(\pi^{1} j_{0}\right) & & \text { on } \Gamma .
\end{aligned}
$$


We remark that the jump of $\pi^{2} j_{0}$ across $\Gamma$ in the last relation is well-defined in the distributional sense because of the control of the two-dimensional curl of $j_{0}$ in (3.14). We recall that $e_{1}$ is normal to $\Gamma_{Y}^{1}$ and $e_{2}$ is tangential to $\Gamma_{Y}^{1}$.

Proof. Step 1. Two-dimensionality of $j_{0}$ and relations (3.10)-(3.13). Equation (3.10) is an immediate consequence of $\nabla_{x} \cdot H^{\eta}=0$ on $\Omega$. The other relations (3.11)-(3.13) are consequences of the Maxwell equation (1.2) for the magnetic field,

$$
\eta \operatorname{curl}_{x} H^{\eta}=\eta\left(-i \omega \varepsilon_{\eta} \varepsilon_{0}\right) E^{\eta}=-i \omega \varepsilon_{0} J^{\eta}
$$

Taking the two-scale limit, we obtain $(3.11)$ as for the electric field $E_{0}(x, \cdot)$. Since $J^{\eta}$ is a curl, it has vanishing divergence $\nabla_{x} \cdot J^{\eta}=0$ on $\Omega$. Passing to the two-scale limit one obtains with a localization in $x$ and an integration by parts

$$
\int_{\Sigma} j_{0}\left(x, y_{1}, y_{2}\right) \cdot \nabla \varphi\left(y_{1}, y_{2}, 0\right) d\left(y_{1}, y_{2}\right)=0
$$

for almost every $x \in \Omega$ and every test function $\varphi \in C_{c}^{\infty}(Y)$. Upon extending $j_{0}$ by zero to all of $Y^{2}$, we may extend the integral in (3.17) also to $Y^{2}$. Using test functions of the form $\varphi\left(y_{1}, y_{2}, y_{3}\right)=\theta\left(y_{1}, y_{2}\right) \phi\left(y_{3}\right)$ in this relation provides

$$
\begin{aligned}
\pi^{3} j_{0}\left(x, y_{1}, y_{2}\right)=0 & \text { for } \quad\left(y_{1}, y_{2}\right) \in \Sigma \\
\nabla_{\left(y_{1}, y_{2}\right)} \cdot \pi^{1,2} j_{0}\left(x, y_{1}, y_{2}\right) & =0 \quad \text { for } \quad\left(y_{1}, y_{2}\right) \in Y^{2}
\end{aligned}
$$

This shows the two-dimensionality of $j_{0}$ and (3.12).

We next verify (3.13), the relation between the jump of the magnetic field and the current in $\Sigma$. We emphasize that this relation should be regarded as an extension of (3.11), since it is concerned with the singular part of $\operatorname{curl}_{y} H_{0}$. We therefore start once more with the Maxwell equation (3.16) for the magnetic field. Using two-scale convergence, we obtain

$$
\operatorname{curl}_{y} H_{0}(x, y)=-i \omega \varepsilon_{0} j_{0}\left(x, y_{1}, y_{2}\right) \mathcal{H}_{\Sigma_{Y}^{2}}^{2}(y)
$$

The two scale limit is performed with the usual choice of test-functions, on the left hand side, $\eta \operatorname{curl}_{x}$ is integrated by parts and the limit provides the product of $H_{0}$ with $\operatorname{curl}_{y}$ of the test-function. On the right hand side, we use the two-scale limit with concentration of (3.3). The result is, in the sense of distributions, (3.20). While the regular part of (3.20) repeats (3.11), the singular part of (3.20) provides the jump condition (3.13).

Step 2. The curl of the current density. We next want to derive relations (3.14)-(3.15), which are consequences of the Maxwell equation (1.1) for the electric field,

$$
\operatorname{curl}_{x} E^{\eta}=i \omega \mu_{0} H^{\eta}
$$

We construct a sequence of oscillating test-functions as follows. We choose an arbitrary function $\theta \in C_{c}^{\infty}(\Sigma \backslash \Gamma ; \mathbb{R})$ and choose for $\phi^{\eta} \in L^{2}\left(\left(-\frac{1}{2},+\frac{1}{2}\right) ; \mathbb{R}\right)$ the characteristic function with $\phi^{\eta}\left(y_{3}\right)=1$ for $y_{3} \in(-\beta \eta, \beta \eta)$ and zero otherwise. We then choose, with an arbitrary localization function $\psi \in C_{c}^{\infty}(\Omega, \mathbb{R})$, the oscillatory function $\varphi_{\eta}(x)=\psi(x) \theta\left(x_{1} / \eta, x_{2} / \eta\right) \phi^{\eta}\left(x_{3} / \eta\right) e_{3}$ as a test-function. This function is pointing in the direction normal to the ring plane. 
Multiplication of (3.21) with $\frac{1}{\eta} \varphi_{\eta}$, we obtain

$$
\begin{aligned}
& \int_{\Omega} \operatorname{curl}_{x} E^{\eta}(x) \frac{1}{\eta} \varphi_{\eta}(x) d x=\int_{\Sigma_{\eta}} \frac{1}{\eta^{2}} E^{\eta}(x) \eta \operatorname{curl}_{x} \varphi_{\eta}(x) d x \\
= & \frac{1}{i \kappa} \int_{\Sigma_{\eta}}\left(J^{\eta}(x)-\eta E^{\eta}(x)\right) \cdot \eta \operatorname{curl}_{x} \varphi_{\eta}(x) d x \\
\rightarrow & -\frac{1}{i \kappa} \int_{\Omega} \psi(x) \int_{\Sigma} j_{0}\left(x, y_{1}, y_{2}\right) \cdot \nabla_{\left(y_{1}, y_{2}\right)}^{\perp} \theta\left(y_{1}, y_{2}\right) d\left(y_{1}, y_{2}\right) d x .
\end{aligned}
$$

In the first equation, we have integrated by parts without boundary terms, since the normal vector of the flat boundaries of the ring is $\pm e_{3}$ and the test-function points in direction $e_{3}$. Along the (thin) lateral boundaries of $\Sigma_{\eta}$, the test-function vanishes. In the second equality, we have inserted the definition of $J^{\eta}$ from (2.1); in the limit process we exploited the property (3.3) of $j_{0}$ and the $L^{2}$-boundedness of $E^{\eta}$.

We now investigate the right hand side of (3.21), tested with $\frac{1}{\eta} \varphi_{\eta}$. We calculate

$$
\begin{aligned}
& i \omega \mu_{0} \frac{1}{\eta} \int_{\Omega} H^{\eta}(x) \varphi_{\eta}(x) d x=i \omega \mu_{0} \frac{1}{\eta} \int_{\Sigma_{\eta}} \pi^{3} H^{\eta}(x) \psi(x) \theta\left(x_{1} / \eta, x_{2} / \eta\right) d x \\
& \rightarrow 2 \beta i \omega \mu_{0} \int_{\Omega} \psi(x) \int_{\Sigma} t r_{\Sigma}^{3} H_{0}\left(x, y_{1}, y_{2}\right) \theta\left(y_{1}, y_{2}\right) d\left(y_{1}, y_{2}\right) d x
\end{aligned}
$$

where we used (2.14) of the trace lemma 2.3 .

We now compare the two expressions of (3.22) and (3.23), which were calculated from the two sides of (3.21). Since $\psi$ was arbitrary, we obtain, for almost every $x \in \Omega$,

$$
-\frac{1}{i \kappa} \int_{\Sigma} j_{0}(x, \cdot) \cdot \nabla_{\left(y_{1}, y_{2}\right)}^{\perp} \theta=2 \beta i \omega \mu_{0} \int_{\Sigma} \operatorname{tr}_{\Sigma}^{3} H_{0} \theta
$$

or, equivalently,

$$
\int_{\Sigma} j_{0}(x, \cdot) \cdot \nabla_{\left(y_{1}, y_{2}\right)}^{\perp} \theta=2 \beta \kappa \omega \mu_{0} \int_{\Sigma} t r_{\Sigma}^{3} H_{0} \theta
$$

Since $\theta$ was arbitrary, we can conclude (3.14).

It remains to derive the jump condition (3.15). We note that with this equation, we determine the singular part of the left hand side of (3.14). Correspondingly, we use the same calculation as for the derivation of (3.14), also with the same construction of the test-function $\varphi_{\eta}$. The only exception is that we now consider an arbitrary test-function $\theta \in C_{c}^{\infty}(\Sigma ; \mathbb{R})$, permitting it to have non-vanishing values along the slit $\Gamma$. We restrict ourselfs to functions $\theta$ that are independent of $y_{1}$ in a neighborhood of $\Gamma$. We observe already here that the calculation of (3.23) remains valid.

In the calculation of (3.22), we obtain one additional term at the point where we insert the definition of $J^{\eta}$, due to contributions in the slit,

$$
\begin{aligned}
& \int_{\Omega} \operatorname{curl}_{x} E^{\eta}(x) \frac{1}{\eta} \varphi_{\eta}(x) d x= \\
& \int_{\Sigma_{\eta}} \frac{\eta}{i \kappa}\left(J^{\eta}(x)-\eta E^{\eta}(x)\right) \cdot \operatorname{curl}_{x} \varphi_{\eta}(x) d x+\int_{\Gamma_{\eta}} \frac{1}{\eta^{3}} J^{\eta}(x) \cdot \eta \operatorname{curl}_{x} \varphi_{\eta}(x) d x .
\end{aligned}
$$

We have to analyze the additional contribution $I_{\eta}$, which we first simplify to

$$
I_{\eta}:=\int_{\Gamma_{\eta}} \frac{1}{\eta^{3}} J^{\eta}(x) \cdot \eta \operatorname{curl}_{x} \varphi_{\eta}(x) d x=\int_{\Gamma_{\eta}} \frac{1}{\eta^{3}} J^{\eta}(x) \cdot e_{1} \partial_{y_{2}} \theta\left(x_{1} / \eta, x_{2} / \eta\right) \psi(x) d x+o(1) .
$$


On the right hand side appears an average of $J^{\eta} \cdot e_{1}$ over the slit, weighted with the fixed function $\partial_{y_{2}} \theta$. To simplify further, we abbreviate with $\Theta: \Sigma \rightarrow \mathbb{R}, \Theta\left(y_{1}, y_{2}\right):=$ $\partial_{y_{2}} \theta\left(y_{1}, y_{2}\right)$, with compact support in $\Sigma$. The integral of interest has now the form

$$
I_{\eta}=\int_{\Gamma_{\eta}} \frac{1}{\eta^{3}} J^{\eta}(x) \cdot e_{1} \Theta\left(x_{1} / \eta, x_{2} / \eta\right) \psi(x) d x+o(1) .
$$

Such slit averages are determined in Lemma 3.3 below. The lemma provides

$$
I_{\eta} \rightarrow 2 \alpha \int_{\Omega} \psi(x) \int_{\Gamma} \pi^{1} j_{0}(x, \cdot) \Theta\left(y_{1}, y_{2}\right) d \mathcal{H}^{1}\left(y_{1}, y_{2}\right) d x
$$

We recall that this determined the additional term, which is introduced into (3.24) by the fact that $\theta$ does not vanish on $\Gamma$. Recalling furthermore $\Theta=\partial_{y_{2}} \theta$, we obtain instead of (3.24) now

$$
-\frac{1}{i \kappa} \int_{\Sigma} j_{0}(x, \cdot) \cdot \nabla_{\left(y_{1}, y_{2}\right)}^{\perp} \theta+2 \alpha \int_{\Gamma} \pi^{1} j_{0}(x, \cdot) \partial_{y_{2}} \theta=2 \beta i \omega \mu_{0} \int_{\Sigma} \operatorname{tr}_{\Sigma}^{3} H_{0} \theta
$$

or, equivalently,

$$
\int_{\Sigma} j_{0}(x, \cdot) \cdot \nabla_{\left(y_{1}, y_{2}\right)}^{\perp} \theta=i \kappa 2 \alpha \int_{\Gamma} \pi^{1} j_{0}(x, \cdot) \partial_{y_{2}} \theta+2 \beta \kappa \omega \mu_{0} \int_{\Sigma} \operatorname{tr}_{\Sigma}^{3} H_{0} \theta .
$$

Since $\theta$ was arbitrary on $\Gamma$, this provides (3.15).

Lemma 3.3 (Slit averages of $\left.J^{\eta} \cdot e_{1}.\right)$. For test-functions $\Theta \in C_{c}^{\infty}(\Sigma ; \mathbb{R})$ and $\psi \in C_{c}^{\infty}(\Omega)$, there holds

$$
\int_{\Gamma_{\eta}} \frac{1}{\eta^{3}} e_{1} \cdot J^{\eta}(x) \Theta\left(x_{1} / \eta, x_{2} / \eta\right) \psi(x) d x \rightarrow 2 \alpha \int_{\Omega} \psi(x) \int_{\Gamma} \pi^{1} j_{0}(x, \cdot) \Theta d \mathcal{H}^{1}\left(y_{1}, y_{2}\right) d x .
$$

Proof. The statement of the lemma is based on two facts: The vanishing divergence $\nabla \cdot J^{\eta}$ allows to evaluate the normal component of the flux through the slit. Additionally, the a priori estimates guarantee that the flux is concentrated in the ring plane.

In this proof, we assume without loss of generality that the slit is centered along a segment $\Gamma_{Y}^{1} \subset\left\{\left(y_{1}, y_{2}, y_{3}\right): y_{1}=y_{3}=0\right\}$, such that the slit in the single cell is $\Gamma_{Y}^{\eta} \subset\left\{\left(y_{1}, y_{2}, y_{3}\right):\left|y_{1}\right|<\alpha \eta^{3},\left|y_{3}\right|<\beta \eta\right\}$. We furthermore assume that $\Theta$ has its support in a neighborhood of the slit, and that $\Theta$ is independent of $y_{1}$ in a (smaller) neighborhood of the slit. Our analysis will contain integrals over the extended slit $S_{\eta}:=\left\{\left(y_{1}, y_{2}, y_{3}\right)\right.$ : $\left.y_{1} \in\left(-\alpha \eta^{3}, \alpha \eta^{3}\right)\right\}$. In order to localize in the ring-plane we use, for $\delta>0$, a family of smooth functions

$$
\phi_{\delta}^{\eta}: \mathbb{R} \rightarrow \mathbb{R}, \quad \phi_{\delta}^{\eta}\left(y_{3}\right)=1 \forall y_{3} \in[-\beta \eta, \beta \eta], \quad \phi_{\delta}^{\eta}\left(y_{3}\right)=0 \text { if }\left|y_{3}\right|>(\beta+\delta) \eta .
$$

In order to derive (3.25), we construct a test-function with a large gradient across the slit. We choose a smooth function $\tilde{\vartheta}:[0,1 / 2) \rightarrow \mathbb{R}$ with $\tilde{\vartheta}(0)=1$ and with compact support, and set

$$
\begin{aligned}
& \vartheta^{\eta}\left(y_{1}\right):= \begin{cases}\left(2 \alpha \eta^{3}\right)^{-1}\left(y_{1}+\alpha \eta^{3}\right) & \text { for }\left|y_{1}\right| \leq \alpha \eta^{3}, \\
\tilde{\vartheta}\left(y_{1}-\alpha \eta^{3}\right) & \text { for } y_{1}>\alpha \eta^{3}, \\
0 & \text { for } y_{1}<-\alpha \eta^{3},\end{cases} \\
& \varphi_{\eta}^{\delta}(x):=\vartheta^{\eta}\left(x_{1} / \eta\right) \phi_{\delta}^{\eta}\left(x_{3} / \eta\right) \Theta\left(x_{1} / \eta, x_{2} / \eta\right) \psi(x) .
\end{aligned}
$$


Using $\eta \varphi_{\eta}^{\delta}$ as a test-function in the relation $\nabla_{x} \cdot J^{\eta}=0$, we obtain

$$
\begin{aligned}
0 & =\int_{\Omega} J^{\eta} \cdot \eta \nabla \varphi_{\eta}^{\delta} \\
& =\int_{S_{\eta}} J^{\eta} \cdot e_{1} \frac{1}{2 \alpha \eta^{3}} \phi_{\delta}^{\eta}\left(x_{3} / \eta\right) \Theta\left(x_{1} / \eta, x_{2} / \eta\right) \psi(x)+\int_{\Omega \backslash S_{\eta}} J^{\eta} \cdot \nabla_{y}\left[\vartheta^{\eta} \phi_{\delta}^{\eta} \Theta\right] \psi(x)+o(1) .
\end{aligned}
$$

The first integral on the right hand side is essentially the integral, that we wish to evaluate, except that we integrate over a region larger than $\Gamma_{\eta}$. For the error term, we claim that

$$
\lim _{\delta \rightarrow 0} \lim _{\eta \rightarrow 0} \int_{S_{\eta} \backslash \Gamma_{\eta}}\left|J^{\eta}\right| \frac{1}{\eta^{3}} \phi_{\delta}^{\eta}\left(x_{3} / \eta\right)=0 .
$$

Indeed, the a priori estimate for $E^{\eta}$ guarantees

$$
\begin{aligned}
\int_{S_{\eta} \backslash \Gamma_{\eta}}\left|J^{\eta}\right| \frac{1}{\eta^{3}} \phi_{\delta}^{\eta}\left(x_{3} / \eta\right) & =\int_{S_{\eta} \backslash \Gamma_{\eta}}\left|E^{\eta}\right| \frac{1}{\eta^{2}} \phi_{\delta}^{\eta}\left(x_{3} / \eta\right) \\
& \leq \frac{1}{\eta^{2}}\left\|E^{\eta}\right\|_{L^{2}(\Omega)}\left|S_{\eta}^{\delta} \backslash \Gamma_{\eta}\right|^{1 / 2} \leq C \frac{1}{\eta^{2}}\left|\delta \eta \cdot \eta^{3}\right|^{1 / 2}=C|\delta|^{1 / 2}
\end{aligned}
$$

where we denoted by $S_{\eta}^{\delta}$ the part of the slit-extension where $\phi_{\delta}^{\eta}\left(x_{3} / \eta\right)$ does not vanish. We have thus obtained (3.29). Similarly, we investigate an error term from the right hand side of (3.28) as

$$
\begin{aligned}
& \int_{\Omega \backslash S_{\eta}}\left|J^{\eta} \cdot e_{3}\right|\left|\partial_{y_{3}} \phi_{\delta}^{\eta}\right| \leq \int_{\Omega \backslash\left(S_{\eta} \cup \Sigma_{\eta}\right)}\left|J^{\eta}\right| \frac{1}{\delta \eta} \mathbf{1}_{\left\{\left|y_{3}\right| \in(\beta \eta,(\beta+\delta) \eta)\right\}} \\
= & \int_{\Omega \backslash\left(S_{\eta} \cup \Sigma_{\eta}\right)}\left|E^{\eta}\right| \frac{1}{\delta} \mathbf{1}_{\left\{\left|y_{3}\right| \in(\beta \eta,(\beta+\delta) \eta)\right\}} \leq\left\|E^{\eta}\right\|_{L^{2}(Q)} \frac{1}{\delta}|\delta \eta|^{1 / 2} .
\end{aligned}
$$

We obtain

$$
\lim _{\delta \rightarrow 0} \lim _{\eta \rightarrow 0} \int_{\Omega \backslash S_{\eta}}\left|J^{\eta}\right|\left\{\left|\partial_{y_{3}} \phi_{\delta}^{\eta}\right|+\left|\phi_{\delta}^{\eta}\right|\right\}=0 .
$$

The error estimates (3.29) and (3.30) allow to conclude from (3.28), by taking the limits $\eta \rightarrow 0$ and then $\delta \rightarrow 0$, the relation

$$
\lim _{\eta \rightarrow 0} \int_{\Gamma_{\eta}} J^{\eta} \cdot e_{1} \frac{1}{\eta^{3}} \Theta\left(x_{1} / \eta, x_{2} / \eta\right) \psi(x)=-2 \alpha \lim _{\delta \rightarrow 0} \lim _{\eta \rightarrow 0} \int_{\Sigma_{\eta}} J^{\eta} \cdot \nabla_{y}\left[\vartheta^{\eta} \Theta\right] \psi(x),
$$

where the right hand side is due to the smallness of

$$
\lim _{\delta \rightarrow 0} \lim _{\eta \rightarrow 0} \int_{[\Sigma \times(-(\beta+\delta) \eta,(\beta+\delta) \eta)] \backslash S_{\eta}} J^{\eta} \cdot \nabla_{y}\left[\vartheta^{\eta} \Theta\right] \psi(x)-\lim _{\delta \rightarrow 0} \lim _{\eta \rightarrow 0} \int_{\Sigma_{\eta}} J^{\eta} \cdot \nabla_{y}\left[\vartheta^{\eta} \Theta\right] \psi(x) .
$$

On the left, we find the integral that we wanted to determine. On the right hand side, due to the factor $\vartheta^{\eta}$, we obtain the limit of integrals over the part $y_{1}>0$ of the ring, hence

$$
\lim _{\eta \rightarrow 0} \int_{\Gamma_{\eta}} J^{\eta} \cdot e_{1} \frac{1}{\eta^{3}} \Theta\left(x_{1} / \eta, x_{2} / \eta\right) \psi(x)=-2 \alpha \int_{\Omega} \psi(x) \int_{\Sigma} j_{0} \cdot \nabla_{y}[\tilde{\vartheta} \Theta] \mathbf{1}_{y_{1}>0}(y) .
$$

With an integration by parts, exploiting that the regular part of $\nabla_{y} \cdot j_{0}$ vanishes, we obtain (3.25). 
Another form of the cell-problem. Since $j_{0}$ has a vanishing two-dimensional divergence, we can write it with a potential $\Phi \in W^{1,2}(\Sigma, \mathbb{C})$ as

$$
j_{0}\left(x, y_{1}, y_{2}\right)=\nabla_{\left(y_{1}, y_{2}\right)}^{\perp} \Phi\left(x, y_{1}, y_{2}\right) .
$$

Since the normal component (with respect to $\Sigma$ ) of $j_{0}$ has no jump, we also have the boundary condition

$$
j_{0}\left(x, y_{1}, y_{2}\right) \cdot \nu_{\Sigma}=0 \quad \text { for }\left(y_{1}, y_{2}\right) \in \partial \Sigma .
$$

Consequently, the potential $\Phi$ satisfies the boundary condition $\nabla_{\left(y_{1}, y_{2}\right)} \Phi \cdot \tau_{\Sigma}=0$ on $\partial \Sigma$, it is constant on the inner and on the outer boundary of $\Sigma$. The magnetic field $H_{0}=H_{0}(x, \cdot)$ and the potential $\Phi=\Phi(x, \cdot)$ satisfy the following set of equations.

$$
\begin{aligned}
\operatorname{div}_{y} H_{0} & =0 \quad \text { in } Y \\
\operatorname{curl}_{y} H_{0} & =0 \quad \text { in } Y \backslash \bar{\Sigma}_{Y}^{2} \\
{\left[\left(\pi^{1,2} H_{0}\right)^{\perp}\right]_{\Sigma} } & =-i \omega \varepsilon_{0} \nabla_{\left(y_{1}, y_{2}\right)}^{\perp} \Phi \\
-\Delta_{\left(y_{1}, y_{2}\right)} \Phi & =2 \kappa \omega \mu_{0} \beta \operatorname{tr}_{\Sigma}^{3} H_{0}+i 2 \alpha \kappa \partial_{y_{2}}^{2} \Phi\left(y_{1}, y_{2}\right) \mathcal{H}_{\Gamma}^{1} \quad \text { on } \Sigma .
\end{aligned}
$$

Representation of the pair $\left(H_{0}(x, y), j_{0}(x, y)\right)$. We will show in the next subsection, that the solution space to the cell problem for $\left(H_{0}, j_{0}\right)$ is four dimensional. This implies that the two-scale limit functions can be written, for appropriate coefficients $h_{k}(x)$, $k=0,1,2,3$, with the help of four basis function pairs. We therefore obtain with the next subsection, in analogy to (3.8) for the electric field, for some fixed cell solutions $\left(H^{k}(y), j^{k}(y)\right)$, the representation

$$
\begin{aligned}
H_{0}(x, y) & =\sum_{k=0}^{3} h_{k}(x) H^{k}(y) \\
j_{0}(x, y) & =\sum_{k=0}^{3} h_{k}(x) j^{k}(y) .
\end{aligned}
$$

This is our aim in the next subsection.

\subsection{The four dimensional space of cell solutions}

In this subsection we construct four basis functions $\left(H^{k}, \Phi^{k}\right), k=0,1,2,3$, which are solutions to the cell problem (3.31)-(3.36). By showing that the construction provides indeed a basis of the solution space, we conclude that the solution space is four dimensional. The aim of this subsection is the following proposition.

Proposition 3.1. The solution space to the cell problem (3.33)-(3.36) is four dimensional. It is spanned by shape functions $\left(H^{k}(y), \Phi^{k}\left(y_{1}, y_{2}\right)\right), k=0,1,2,3$, which are uniquely determined as the solution of (3.33)-(3.36) with the normalization

$$
\begin{aligned}
& \int_{Y} H^{k}=e_{k},\left.\quad \Phi^{k}\right|_{\partial \Sigma}=0 \text { for } k \in\{1,2,3\}, \\
& \int_{Y} H^{0}=0, \quad-\left.i \omega \varepsilon_{0} \Phi^{0}\right|_{\partial_{o} \Sigma}=1,\left.\Phi^{0}\right|_{\partial_{i} \Sigma}=0 .
\end{aligned}
$$


The construction of solutions is based on the Lemma of Lax-Milgram in an appropriate function space. In order to transform the problem into such a setting, we consider the following space $X$,

$$
\begin{gathered}
X:=\left\{u \in L_{\mathrm{per}}^{2}\left(Y, \mathbb{C}^{3}\right) \mid \operatorname{div} u \in L^{2}(Y), \operatorname{curl} u=0 \text { in } Y \backslash \bar{\Sigma}_{Y}^{2},\right. \\
\left.\left[\pi^{1,2} u\right]_{\Sigma} \in L^{2}(\Sigma), \operatorname{tr}_{\Gamma}\left(\left[\pi^{2} u\right]_{\Sigma}\right) \in L^{2}(\Gamma)\right\},
\end{gathered}
$$

equipped with the norm

$$
\|u\|_{X}^{2}:=\|u\|_{L^{2}(Y)}^{2}+\|\operatorname{div} u\|_{L^{2}(Y)}^{2}+\left\|\left[\pi^{1,2} u\right]_{\Sigma}\right\|_{L^{2}(\Sigma)}^{2}+\left\|r_{\Gamma}\left(\left[\pi^{2} u\right]_{\Sigma}\right)\right\|_{L^{2}(\Gamma)}^{2} .
$$

The function $u$ will represent, later on, the magnetic field $H_{0}$. Some comments should be made concerning the definition of the space $X$. The condition curl $u=0$ in $Y \backslash \bar{\Sigma}_{Y}^{2}$ is well-defined in the distributional sense; it furthermore justifies the evaluation of tangential traces on $\Sigma$. The jump condition $\left[\pi^{1,2} u\right]_{\Sigma}$ then means that the jump of the distributional traces can be represented by a function in $L^{2}(\Sigma)$.

In order to give sense to the last condition in the definition of $X$, we have to give a meaning to the distribution $\operatorname{tr}_{\Gamma}\left(\left[\pi^{2} u\right]_{\Sigma}\right)$, the trace on $\Gamma$ of the second component. For notational convenience we assume again $\Gamma \subset\left\{y_{1}=0\right\}$. We choose a definition of the trace (as a distribution), which is consistent with the formula $\partial_{1} u_{2}=\partial_{2} u_{1}$. We set, for $\varphi \in C_{c}^{\infty}(\Sigma)$

$$
\left\langle\operatorname{tr}_{\Gamma}\left(\left[\pi^{2} u\right]_{\Sigma}\right), \varphi\right\rangle_{\Gamma}:=\int_{\Sigma \cap\left\{y_{1}>0\right\}} u_{2} \partial_{1} \varphi-u_{1} \partial_{2} \varphi .
$$

We note two facts about this definition. 1. The definition is independent of the values of $\varphi$ outside $\Gamma$. 2. The trace from the side $y_{1}<0$ is identical to the trace from $y_{1}>0$. Both facts 1. and 2. follow from the definition of the trace on $\Sigma$. For $\phi \in C_{c}^{\infty}\left(\left(-\frac{1}{2}, \frac{1}{2}\right), \mathbb{R}\right)$ and $\phi\left(y_{3}=0\right)=1$ there holds (formally, we exploit $\partial_{3} u_{2}=\partial_{2} u_{3}$ and $\partial_{3} u_{1}=\partial_{1} u_{3}$ ),

$$
\int_{\Sigma} u_{2} \partial_{1} \varphi-u_{1} \partial_{2} \varphi=\int_{\Sigma \times(0,1 / 2)} u_{2} \partial_{3} \phi \partial_{1} \varphi-u_{3} \phi \partial_{2} \partial_{1} \varphi-u_{1} \partial_{3} \phi \partial_{2} \varphi+u_{3} \phi \partial_{1} \partial_{2} \varphi=0
$$

the last equality being due to $\operatorname{curl} u=0$ outside $\Sigma$.

Since we want to employ the Lax-Milgram lemma to find solutions, we next define a sesquilinear form on $X$. We introduce the number $k_{0}>0$ through $k_{0}^{2}=\varepsilon_{0} \mu_{0} \omega^{2}$ and define the sesquilinear form $b: X \times X \rightarrow \mathbb{C}$ as

$$
\begin{aligned}
b(u, v):= & \int_{Y} \operatorname{div} u \operatorname{div} \bar{v}-i 2 \beta k_{0}^{2} \int_{Y} u \cdot \bar{v}+\frac{1}{\kappa} \int_{\Sigma}\left[\pi^{1,2} u\right]_{\Sigma}\left[\pi^{1,2} \bar{v}\right]_{\Sigma} \\
& +2 \alpha i \int_{\Gamma} \operatorname{tr}_{\Gamma}\left(\left[\pi^{2} u\right]_{\Sigma}\right) \operatorname{tr}_{\Gamma}\left(\left[\pi^{2} \bar{v}\right]_{\Sigma}\right) .
\end{aligned}
$$

The form $b$ is continuous on $X \times X$. We claim that, for every anti-linear form $\langle f,\rangle:. X \rightarrow$ $\mathbb{C}$, there holds

$$
\exists ! \quad u \in X: \quad b(u, v)=\langle f, v\rangle \quad \forall v \in X .
$$

The existence result of (3.45) is a consequence of the Lax-Milgram lemma, but we have to construct a modified bilinear form in order to satisfy a coercivity property. We consider the sesquilinear form

$$
\tilde{b}(u, v):=b(u, v)+\left(\int_{Y} u\right)\left(\int_{Y} \bar{v}\right) .
$$


We claim that, for a sufficiently small angular variable $\varpi>0$, there exists a positive constant $c_{c}>0$, such that the rotated bilinear form satisfies

$$
\begin{aligned}
& \operatorname{Re}\left[e^{-\varpi i \tilde{b}}(u, u)\right]=(\cos \varpi) \int_{Y}|\operatorname{div} u|^{2}-(\sin \varpi) 2 \beta k_{0}^{2} \int_{Y}|u|^{2} \\
& \quad+(\cos \varpi) \frac{1}{\kappa} \int_{\Sigma}\left|\left[\left(\pi^{1,2} u\right)\right]_{\Sigma}\right|^{2}+(\sin \varpi) 2 \alpha \int_{\Gamma}\left|t_{\Gamma}\left(\left[\pi^{2} u\right]_{\Sigma}\right)\right|^{2}+(\cos \varpi)\left|\int_{Y} u\right|^{2} \\
& \geq c_{c}\|u\|_{X}^{2} .
\end{aligned}
$$

Let us sketch the argument for the positivity property (3.46). The only negative term is the second integral, and the other terms are equivalent to the norms. We therefore only have to justify that the second term is, in absolute value, bounded by the other integrals for sufficiently small $\varpi>0$. This can be seen with a contradiction argument. Assume that there exists a sequence of functions $u_{k} \in X$ with the normalization $\left\|u_{k}\right\|_{L^{2}(Y)}=1$ which satisfies at the same time $\operatorname{div} u_{k} \rightarrow 0$ in $L^{2}(Y),\left[\left(\pi^{1,2} u_{k}\right)\right]_{\Sigma} \rightarrow 0$ in $L^{2}(\Sigma)$, and $\int_{Y} u \rightarrow 0$. Then every weak limit $u$ of a subsequence $k \rightarrow \infty$ has vanishing divergence in $Y$, vanishing curl in $Y \backslash \Sigma_{Y}^{2}$, vanishing jump across $\Sigma_{Y}^{2}$, and vanishing average. This implies that the limit $u$ is constant in $Y$. The compensated compactness lemma can be applied to $u_{k}$ on $Y$ and implies the strong convergence $u_{k} \rightarrow u=0$ in $L^{2}(Y)$, in contradiction to the normalization of $u_{k}$. The contradiction shows (3.46).

At this point, the Lax-Milgram lemma implies that, for every $\tilde{f} \in X^{*}$, there exists a unique solution of $\tilde{b}(u, v)=\langle\tilde{f}, v\rangle \forall v \in X$. In order to show the existence statement of (3.45), we argue as follows. Given $f \in X^{*}$, we construct

$$
\langle\tilde{f}, v\rangle:=\langle f, v\rangle-\frac{1}{i 2 \beta k_{0}^{2}} \sum_{k=1}^{3}\left\langle f, e_{k}\right\rangle \cdot\left\langle e_{k}, v\right\rangle .
$$

We claim that the solution $u \in X$ of $\tilde{b}(u,)=.\langle\tilde{f},$.$\rangle provides the desired solution of (3.45).$ Indeed, inserting the test-function $v=e_{k}$, we obtain $\tilde{b}\left(u, e_{k}\right)=\left\langle\tilde{f}, e_{k}\right\rangle$, hence

$$
\left(1-i 2 \beta k_{0}^{2}\right) \int_{Y} u \cdot e_{k}=\left(1-\frac{1}{i 2 \beta k_{0}^{2}}\right)\left\langle f, e_{k}\right\rangle
$$

and thus $\int_{Y} u \cdot e_{k}=-\left(i 2 \beta k_{0}^{2}\right)^{-1}\left\langle f, e_{k}\right\rangle$. We conclude that $\tilde{b}(u, v)-b(u, v)=\langle\tilde{f}, v\rangle-\langle f, v\rangle$, we have therefore solved with $u$ equation (3.45). A similar calulation shows that solutions of (3.45) are also solutions of $\tilde{b}(u, v)=\langle\tilde{f}, v\rangle \forall v \in X$, which implies the uniqueness statement of (3.45).

In the following, the form $f$ will be chosen either as a function with support away from the ring,

$$
\langle f, v\rangle:=\int_{Y} g \cdot \bar{v} \forall v \in X, \quad \text { where } g \in L^{2}\left(Y, \mathbb{C}^{3}\right), \operatorname{div} g=0, \operatorname{supp} g \subset Y \backslash \bar{\Sigma}_{Y}^{2},
$$

or we choose the special form $f=f_{0}$,

$$
\left\langle f_{0}, v\right\rangle:=\int_{\Sigma} \nabla_{\left(y_{1}, y_{2}\right)} \theta_{0} \cdot\left[\pi^{1,2} \bar{v}\right]_{\Sigma}, \forall v \in X,
$$

where $\theta_{0} \in H^{1}(\Sigma)$ is the unique solution of

$$
\begin{aligned}
\Delta_{\left(y_{1}, y_{2}\right)} \theta_{0} & =0 \quad \text { on } \Sigma, \\
\left.\theta_{0}\right|_{\partial_{o} \Sigma} & =1,\left.\quad \theta_{0}\right|_{\partial_{i} \Sigma}=0 .
\end{aligned}
$$


We emphasize that, in both cases (3.47) and (3.48), the distribution $f$ has a vanishing divergence in the sense that it vanishes on functions in $v \in X \cap C_{\text {per }}^{\infty}\left(Y ; \mathbb{C}^{3}\right)$, which are gradients. For $f$ as in (3.47), this follows from the fact that the divergence of $g$ vanishes. For $f$ as in (3.48), it is a consequence of the fact that the jumps $\left[\pi^{1,2} \bar{v}\right]_{\Sigma}$ vanish for such test-functions.

We have the following lemma. We use here the vector $\int_{Y} f \in \mathbb{C}^{3}$ defined by $e_{j} \cdot \int_{Y} f:=$ $\left\langle f, e_{j}\right\rangle$ for unit vectors $e_{j} \in \mathbb{C}^{3}$.

Lemma 3.4 (Solution property). Let $f \in X^{*}$ be either as in (3.47) or as in (3.48). Let $u \in X$ be the unique solution to the variational equation $b(u, v)=\langle f, v\rangle$ for all $v \in X$. Then the following holds.

1. There exists a unique potential $\Phi \in H^{1}(\Sigma)$ with $\left.\Phi\right|_{\partial_{i} \Sigma}=0$ such that $-i \omega \varepsilon_{0} \nabla \Phi=$ $\left[\pi^{1,2} u\right]_{\Sigma}$.

2. Setting $H_{0}:=u$ and with $\Phi$ as in 1., the pair $\left(H_{0}, \Phi\right)$ solves (3.33)-(3.36).

3. There holds $-i 2 \beta k_{0}^{2} \int_{Y} H_{0}=\int_{Y} f$.

Proof. Item 1. We have seen already in (3.44), that the two-dimensional curl of traces of $\pi^{1,2} u$ on $\Sigma$ vanishes, hence it vanishes on all of $Y^{2}$. Therefore, there exists a potential $\tilde{\Phi} \in H^{1}(\Sigma)$ with $\nabla \tilde{\Phi}=\left[\pi^{1,2} u\right]$. Since $\tilde{\Phi}$ is constant outside $\Sigma$, it is constant on the inner and on the outer boundary. We fix the value on the inner boundary $\partial_{i} \Sigma$ to be 0 . This determines an additive constant and we obtain $\Phi$ with a normalization in a unique way from $\tilde{\Phi}$.

Item 2. The solution property (3.34) is implemented in the space $X$, see (3.41). Property (3.35) was obtained in Item 1. It remains to verify (3.33) and (3.36).

Verification of (3.33). We want to show for the $L^{2}(Y)$-function $w:=\operatorname{div} u$ the property $w=0$. This is done by an appropriate choice of a test-function in the relation $b(u, v)=$ $\langle f, v\rangle$. We choose the curl-free test function $v:=\nabla \Psi$, where $\Psi \in H_{\mathrm{per}}^{1}(Y)$ is the solution of the Helmholtz problem $\Delta \Psi+i 2 \beta k_{0}^{2} \Psi=w$. Then $v$ is an admissible test function: it satisfies $\operatorname{div} v=\Delta \Psi=w-i k_{0}^{2} \Psi \in L_{\text {per }}^{2}(Y)$ and, as a gradient, it has a vanishing curl.

As already noted above, there holds $\langle f, v\rangle=0$, since $v$ is a gradient. We can therefore calculate

$$
\begin{aligned}
0= & b(u, v)=\int_{Y} \operatorname{div} u \Delta \bar{\Psi}-i 2 \beta k_{0}^{2} \int_{Y} u \cdot \nabla \bar{\Psi} \\
& +\frac{1}{\kappa} \int_{\Sigma}\left[\left(\pi^{1,2} u\right)\right]_{\Sigma}\left[\left(\pi^{1,2} \nabla \bar{\Psi}\right)\right]_{\Sigma}+2 \alpha i \int_{\Gamma} \operatorname{tr}_{\Gamma}\left(\left[\pi^{2} u\right]_{\Sigma}\right) \operatorname{tr}_{\Gamma}\left(\left[\partial_{y_{2}} \bar{\Psi}\right]_{\Sigma}\right) \\
= & \int_{Y} \operatorname{div} u\left(\Delta \bar{\Psi}+i 2 \beta k_{0}^{2} \bar{\Psi}\right)=\int_{Y}|w|^{2} .
\end{aligned}
$$

In the third equality we exploited once more that jumps of $\Psi$ vanish; we performed an integration by parts in the second integral. We conclude that $w:=\operatorname{div} u$ vanishes, hence (3.33) is verified.

Verification of (3.36). We construct a function $\varphi(y)=\theta\left(y_{1}, y_{2}\right) \phi\left(y_{3}\right)$ with $\theta \in C_{c}^{\infty}(\Sigma, \mathbb{C})$ arbitrary, and $\tilde{\phi} \in C_{c}^{\infty}\left(\left[0, \frac{1}{2}\right), \mathbb{R}\right)$ with $\tilde{\phi} \equiv 1$ in a neighborhood of $y_{3}=0$. Extending $\tilde{\phi}$ trivially to a (discontinuous) function $\phi:\left(-\frac{1}{2}, \frac{1}{2}\right) \rightarrow \mathbb{R}$, we finally set $v=\nabla^{r e g} \varphi$, where $\nabla^{r e g} \varphi$ denotes the regular part (with respect to the Lebesgue measure) of $\nabla \varphi$. 
We claim that $v$ is an admissible test-function, i.e. $v \in X$. Indeed, as a gradient, $v$ has a vanishing curl. Furthermore, due to $v \cdot e_{3}=0$ in a neighborhood of $\Sigma$, there holds $\operatorname{div} v \in L^{2}(Y)$. The jumps across $\Sigma$ are smooth functions on $\Sigma$, hence $v \in X$ is satisfied.

We now evaluate both sides of $b(u, v)=\langle f, v\rangle$ for the special test-function $v$. We insert the jump $\left[\pi^{1,2} v\right]_{\Sigma}=\nabla_{\left(y_{1}, y_{2}\right)} \theta$ and obtain from the definition of $b$, exploiting $\operatorname{div} u=0$ from the last step,

$$
\begin{aligned}
b(u, v) & =\int_{Y} \operatorname{div} u\left(\Delta \bar{\varphi}+i 2 \beta k_{0}^{2} \bar{\varphi}\right)+i 2 \beta k_{0}^{2} \int_{\Sigma} u \cdot e_{3} \operatorname{tr}_{\Sigma+} \bar{\varphi} \\
& +\frac{1}{\kappa} \int_{\Sigma}\left[\left(\pi^{1,2} u\right)\right]_{\Sigma}\left[\left(\pi^{1,2} \bar{v}\right)\right]_{\Sigma}+2 \alpha i \int_{\Gamma} \operatorname{tr}_{\Gamma}\left[\pi^{2} u\right]_{\Sigma} \operatorname{tr}_{\Gamma}\left[\pi^{2} \bar{v}\right]_{\Sigma} \\
& =i 2 \beta k_{0}^{2} \int_{\Sigma} u \cdot e_{3} \bar{\theta}-\frac{i \omega \varepsilon_{0}}{\kappa} \int_{\Sigma} \nabla_{\left(y_{1}, y_{2}\right)} \Phi \nabla_{\left(y_{1}, y_{2}\right)} \bar{\theta}+2 \alpha \omega \varepsilon_{0} \int_{\Gamma} \partial_{y_{2}} \Phi \partial_{y_{2}} \bar{\theta}
\end{aligned}
$$

We next evaluate the right hand side of $b(u, v)=\langle f, v\rangle$. For $f \in X^{*}$ as in (3.47), $\langle f, v\rangle=0$ is satisfied for gradients $v$, since $g$ has vanishing divergence and is supported away from $\Sigma$. For $f=f_{0}$ as in (3.48), we calculate

$$
\left\langle f_{0}, v\right\rangle=\int_{\Sigma} \nabla_{\left(y_{1}, y_{2}\right)} \theta_{0}\left[\pi^{1,2} \bar{v}\right]_{\Sigma}=\int_{\Sigma} \nabla_{\left(y_{1}, y_{2}\right)} \theta_{0} \nabla_{\left(y_{1}, y_{2}\right)} \bar{\theta}\left(y_{1}, y_{2}\right)=0
$$

by the choice of $\theta_{0}$ in (3.49) with $\Delta_{\left(y_{1}, y_{2}\right)} \theta_{0}=0$ on $\Sigma$. We obtained $b(u, v)=\langle f, v\rangle=0$, i.e.

$$
\begin{aligned}
& i 2 \beta k_{0}^{2} \int_{\Sigma} u \cdot e_{3} \theta-\frac{i \omega \varepsilon_{0}}{\kappa} \int_{\Sigma} \nabla_{\left(y_{1}, y_{2}\right)} \Phi \nabla_{\left(y_{1}, y_{2}\right)} \theta+2 \alpha \omega \varepsilon_{0} \int_{\Gamma} \partial_{y_{2}} \Phi \partial_{y_{2}} \theta=0 \\
\Leftrightarrow & \int_{\Sigma} \nabla_{\left(y_{1}, y_{2}\right)} \Phi \nabla_{\left(y_{1}, y_{2}\right)} \theta=2 \beta \kappa \omega \mu_{0} \int_{\Sigma} u \cdot e_{3} \theta-i 2 \alpha \kappa \int_{\Gamma} \partial_{y_{2}} \Phi \partial_{y_{2}} \theta .
\end{aligned}
$$

Since $\theta$ was an arbitrary function on $\Sigma$, this shows (3.36). We have therefore concluded that the pair $\left(H_{0}, \Phi\right)$ solves the cell problem (3.33)-(3.36).

Item 3. The normalization can be calculated directly with the unit vector $e_{j}, j=$ $1,2,3$, as

$$
\int_{Y} f \cdot e_{j}=\left\langle f, e_{j}\right\rangle=b\left(u, e_{j}\right)=-i 2 \beta k_{0}^{2} \int_{Y} u \cdot e_{j}
$$

This was the claim.

Proof of Proposition 3.1. We have shown in Lemma 3.4 that some functions $u \in X$ have the following property: setting $H_{0}:=u$ and solving $-i \omega \varepsilon_{0} \nabla \Phi=\left[\pi^{1,2} H_{0}\right]_{\Sigma}$ and $\left.\Phi\right|_{\partial_{i} \Sigma}=0$ for $\Phi$, we obtain a solution $\left(H_{0}, \Phi\right)$ of the cell-problem (3.33)-(3.36). On the other hand, every cell-problem solution $\left(H_{0}, \Phi\right)$ provides with $u:=H_{0}$ such a member of $X$. In this sense, cell-problem solutions can be identified with elements of $X$.

In the following, we denote by $\mathcal{V} \subset X$ subset of all functions $u \in X$ that provide a solution $\left(H_{0}, \Phi\right)$ of the cell-problem. Proposition 3.1 states that the solution space is four dimensional. This is shown once that we prove that the linear map

$$
\mathcal{L}: \mathcal{V} \ni u \mapsto\left(\int_{Y} u \cdot e_{1}, \int_{Y} u \cdot e_{2}, \int_{Y} u \cdot e_{3},\left.\Phi\right|_{\partial_{o} \Sigma}\right) \in \mathbb{C}^{4} \quad \text { is one to one. }
$$


With the last entry, we mean the value of the constant function $\Phi$ on the outer ring boundary. Actually, property (3.50) yields also that the normalization of the proposition can be used to identify the basis functions.

Step 1. Injectivity of $\mathcal{L}$. We show the following statement. Every solution $u \in \mathcal{V}$, which satisfies

$$
\int_{Y} u=0,\left.\quad \Phi\right|_{\partial_{o} \Sigma}=\left.\Phi\right|_{\partial_{i} \Sigma}=0
$$

vanishes idenically. To this end, let $u \in \mathcal{V}$ be such a function.

Because of $\operatorname{div} u=0$ and normalization (3.51), we can write $u$ as the curl of a periodic function $\psi \in H_{\mathrm{per}}^{1}\left(Y, \mathbb{C}^{3}\right), u=\operatorname{curl} \psi$. We use $\psi$ as a test-function in (3.34) and obtain

$$
\begin{aligned}
& 0 \stackrel{(3.34)}{=} \int_{Y \backslash \bar{\Sigma}_{Y}^{2}} \operatorname{curl} u \cdot \bar{\psi}=\int_{Y \backslash \bar{\Sigma}_{Y}^{2}} u \cdot \operatorname{curl} \bar{\psi}-\int_{\Sigma}\left[e_{3} \wedge u\right]_{\Sigma} \cdot \bar{\psi} \\
& =\|u\|_{L^{2}(Y)}^{2}-\int_{\Sigma}\left[\left(\pi^{1,2} u\right)^{\perp}\right]_{\Sigma} \cdot \pi^{1,2} \bar{\psi} \stackrel{(3.35)}{=}\|u\|_{L^{2}(Y)}^{2}+i \omega \varepsilon_{0} \int_{\Sigma} \nabla_{\left(y_{1}, y_{2}\right)}^{\perp} \Phi \cdot \pi^{1,2} \bar{\psi} \\
& \stackrel{(3.51)}{=}\|u\|_{L^{2}(Y)}^{2}-i \omega \varepsilon_{0} \int_{\Sigma} \Phi \nabla_{\left(y_{1}, y_{2}\right)}^{\perp} \cdot\left(\pi^{1,2} \bar{\psi}\right)=\|u\|_{L^{2}(Y)}^{2}-i \omega \varepsilon_{0} \int_{\Sigma} \Phi \pi^{3} \bar{u} \\
& \stackrel{(3.36)}{=}\|u\|_{L^{2}(Y)}^{2}-\frac{i \varepsilon_{0}}{2 \beta \kappa \mu_{0}} \int_{\Sigma} \nabla_{\left(y_{1}, y_{2}\right)} \Phi \nabla_{\left(y_{1}, y_{2}\right)} \bar{\Phi}-\frac{\varepsilon_{0} \alpha}{\beta \mu_{0}} \int_{\Gamma} \partial_{y_{2}} \Phi \partial_{y_{2}} \bar{\Phi}
\end{aligned}
$$

Consequently, $\left\|\nabla_{\left(y_{1}, y_{2}\right)} \Phi\right\|_{L^{2}(\Sigma)}=0$ and therefore also the last (negative) term vanishes. Hence, $\|u\|_{L^{2}(Y)}^{2}=0$, which concludes the injectivity part.

Step 2. Surjectivity of $\mathcal{L}$. In order to show surjectivity of $\mathcal{L}$, we apply Lemma 3.4 with four special elements $f_{0}, f_{1}, f_{2}, f_{3}$, such that the corresponding solutions $U_{0}, U_{1}, U_{2}, U_{3}$ have the desired properties. For $k \in\{1,2,3\}$ we choose $\left\langle f_{k}, v\right\rangle=-i 2 \beta k_{0}^{2} \int_{Y} g_{k} \cdot \bar{v}$, where $g_{k}$ is a divergence-free $L^{2}(Y)$-function vanishing on $\Sigma$, with $\int_{Y} g_{k}=e_{k}$. For instance, for $k \in\{1,2,3\}$, we may take $g_{k}$ to be compactly supported in a small cylinder with principal axis $\Gamma_{l}$ and constant in the direction $e_{k}$ where, with $R=1 / 2-\delta$ close to $1 / 2$ and $J=(-1 / 2,1 / 2)$,

$$
\Gamma_{1}=J \times\{R\} \times\{R\}, \quad \Gamma_{2}=\{R\} \times J \times\{R\}, \quad \Gamma_{2}=\{R\} \times\{R\} \times J,
$$

$\delta>0$ being so small that $\Gamma_{l} \cap \bar{\Sigma}=\emptyset$. The remaining element $f_{0}$ is the one of (3.48). It remains to study the properties of the corresponding solutions $U_{k}$.

By item 3 of Lemma 3.4, there holds $-i 2 \beta k_{0}^{2} \int_{Y} U_{k}=\int_{Y} f_{k}$. For the special distribution $f_{0}$, the average is $\left\langle f_{0}, 1\right\rangle=0$, since constant test-functions $v:=e_{j}$ have vanishing jumps across $\Sigma$. By construction, the other averages are $\left\langle f_{k}, e_{j}\right\rangle=-i 2 \beta k_{0}^{2} \int_{Y} g_{k} \cdot e_{j}=-i 2 \beta k_{0}^{2} \delta_{k, j}$. This shows that the functions $H^{k}:=U_{k}$ are normalized as requested in (3.39)-(3.40).

It remains to verify that $U_{0}$ does not vanish identically. Once this is shown, we have obtained four linearly independent solutions in $\mathcal{V}$. The remaining normalizations of (3.40) can then be achieved by subtracting appropriate multiples of $U_{0}$ from $U_{k}$.

To check this last point, we consider a test function $v \in X$ similar to one in the proof of Lemma 3.4. We set $\varphi(y)=\theta_{0}\left(y_{1}, y_{2}\right) \phi\left(y_{3}\right)$, where $\tilde{\phi} \in C_{c}^{\infty}\left(\left[0, \frac{1}{2}\right), \mathbb{R}\right)$ satisfies $\tilde{\phi} \equiv 1$ in a neighborhood of $y_{3}=0$ and is extended trivially to a (discontinuous) function $\phi:\left(-\frac{1}{2}, \frac{1}{2}\right) \rightarrow \mathbb{R}$. The function $\theta_{0}$ is the special function from the definition of $f_{0}$. We use $v=\nabla^{r e g} \varphi$ as a test-function and obtain $b\left(U_{0}, v\right)=\left\langle f_{0}, v\right\rangle=\int_{\Sigma}\left|\nabla_{\left(y_{1}, y_{2}\right)} \theta_{0}\right|^{2}>0$. This shows that $U_{0}$ cannot vanish identically, and proves the proposition. 


\section{Slit-analysis and derivation of effective equations}

\subsection{A relation for the strength of the ring-current}

In the last section we have identified four cell-problem solutions $H^{k}(y), k \in\{0,1,2,3\}$, such that, for some coefficients $h_{k}(x)$, the cell solution $H_{0}(x, y)$ has necessarily the form $H_{0}(x, y)=\sum_{k=0}^{3} h_{k}(x) H^{k}(y)$, see (3.37). In this section we will establish an additional linear relation between the components $h_{k}(x)$ namely

$$
h_{0}(x)=\sum_{k=1}^{3} \lambda_{k} h_{k}(x)
$$

for almost every $x \in \Omega$. Relation (4.1) establishes a connection between the averaged magnetic field (related to the factors $h_{1}(x), \ldots, h_{3}(x)$ ) and the strength of the ring current (related to the factor $h_{0}(x)$ ). The coefficients $\lambda_{k}$ are introduced below, they are frequency dependent. This fact is due to the physical background of the appearance of the ring current: the ring is in resonance with the electromagnetic field. As a result, moderate averaged magnetic fields $h_{1}(x), \ldots, h_{3}(x)$ can create large ring currents and a large amplitude factor $h_{0}(x)$ in (4.1). The derivation of that formula is based on a careful analysis of the field in the ring and in the slit.

Based on the four cell-problem solutions $H^{k}(y)$, we first define the eight constants $D_{k}^{H}$, $D_{k}^{J} \in \mathbb{C}$ for $k \in\{0,1,2,3\}$. The construction is such that the constants $D_{k}^{H}$ measure the magnetic field $H^{k}(y)$ through the ring, the constants $D_{k}^{J}$ measure the current in the ring.

We choose a weight function $\theta_{Y}: Y^{2} \rightarrow[0,1]$ with the following properties. We recall the notation that the inner simply connected component of $Y^{2} \backslash \Sigma$ was denoted as $\Sigma^{\text {inn }}$, we use here the notation $\Sigma_{c}:=\Sigma \cup \Sigma^{\text {inn }}$ for the ring together with the interior part. The set $\Sigma_{c}$ is an open subset of $Y^{2}=\left(-\frac{1}{2}, \frac{1}{2}\right)^{2}$, possibly convex, necessarily connected and simply connected. There holds $\partial \Sigma_{c}=\partial_{o} \Sigma$. We demand

$$
\begin{aligned}
& \theta_{Y} \in C^{0,1}\left(Y^{2}\right), \quad \operatorname{supp}\left(\theta_{Y}\right) \subset \bar{\Sigma}_{c},\left.\quad \theta_{Y}\right|_{\partial \Sigma_{c}}=0,\left.\quad \theta_{Y}\right|_{\Sigma^{\text {inn }}} \equiv 1, \\
& \nabla \theta_{Y} \equiv\left(\begin{array}{c}
0 \\
-1 / L(\Gamma)
\end{array}\right) \text { in a neighborhood of } \Gamma
\end{aligned}
$$

Property (4.3) expresses that $\theta_{Y}$ is affine in a small neighborhood of $\Gamma$. The value of the (constant) gradient is then determined by the boundary condition on outer and inner boundary.

With this weight function, we define constants $D_{k}^{H}, D_{k}^{J}$ from the four cell-solutions as

$$
\begin{aligned}
D_{k}^{H} & :=\int_{\Sigma_{c}} e_{3} \cdot H^{k}\left(y_{1}, y_{2}, 0\right) \theta_{Y}\left(y_{1}, y_{2}\right) d y_{1} d y_{2} \\
D_{k}^{J} & :=\int_{\Sigma} \nabla_{\left(y_{1}, y_{2}\right)} \theta_{Y}\left(y_{1}, y_{2}\right) \cdot\left(-i \omega \varepsilon_{0}\right) \nabla_{\left(y_{1}, y_{2}\right)} \Phi^{k}\left(y_{1}, y_{2}\right) d y_{1} d y_{2} .
\end{aligned}
$$

Lemma 4.1 (Electric field in the slit I). Let the two-scale limit of $H^{\eta}$ be characterized with coefficients $h_{k}(x)$ as in (3.37). Then, for an arbitrary localization function $\psi \in C_{c}^{\infty}(\Omega)$, there holds

$$
\lim _{\eta \rightarrow 0} \frac{1}{\eta^{2} L(\Gamma)} \int_{\Gamma_{\eta}} \pi^{1} E^{\eta}(x) \psi(x) d x=\int_{\Omega} \psi(x) \sum_{k=0}^{3}\left(-2 i \beta \omega \mu_{0} D_{k}^{H}-\frac{1}{\omega \varepsilon_{0} \kappa} D_{k}^{J}\right) h_{k}(x) d x,
$$


We recall the relation $\nabla \Phi^{k}=-\left(j^{k}\right)^{\perp}$, which shows that $D_{k}^{J}$ is a measure for the current of the $k$-th basis function of the cell problem. The lemma is a consequence of the Maxwell equation for the electric field: the Stokes formula relates the electric field in ring and slit with the magnetic field through the ring.

Proof. We start from (1.1), the relation curl $E^{\eta}=i \omega \mu_{0} H^{\eta}$. The proof of the lemma consists in the choice of an appropriate test-function in this relation. We choose $\varphi_{\eta}(x):=$ $\varphi^{\eta}(x / \eta)$, where $\varphi^{\eta}$ is defined by the weight function $\theta_{Y}$ and a characteristic function in the ring as $\varphi^{\eta}(y):=\theta_{Y}\left(y_{1}, y_{2}\right) \chi_{(-\beta \eta, \beta \eta)}\left(y_{3}\right) e_{3}$. We use the test-function $\frac{1}{\eta} \varphi_{\eta}(x) \psi(x)$ in the Maxwell equation and obtain, for the left hand side,

$$
\begin{aligned}
& \frac{1}{\eta} \int_{\Omega} \operatorname{curl} E^{\eta}(x) \cdot \varphi_{\eta}(x) \psi(x) d x=\frac{1}{\eta} \int_{\Omega} E^{\eta}(x) \cdot \operatorname{curl}\left(\varphi_{\eta} \psi\right)(x) d x \\
& =-\frac{1}{\eta^{2}} \int_{\Sigma_{\eta}} \pi^{1,2} E^{\eta}(x) \cdot \nabla_{\left(y_{1}, y_{2}\right)}^{\perp} \theta_{Y}\left(\frac{x_{1}}{\eta}, \frac{x_{2}}{\eta}\right) \psi(x) d x-\frac{1}{\eta^{2} L(\Gamma)} \int_{\Gamma_{\eta}} \pi^{1} E^{\eta}(x) \psi(x) d x+o(1) .
\end{aligned}
$$

In the last line we have used the fact that $\theta_{Y}$ is constant in $\Sigma^{i}$ and that $\nabla_{\left(y_{1}, y_{2}\right)} \theta_{Y}\left(y_{1}, y_{2}\right)=$ $(0,-1 / L(\Gamma))^{T}$ for $\left(y_{1}, y_{2}\right) \in \Gamma^{\eta}$. The last integral coincides with the left hand side of (4.6). We calculate for the other integral

$$
\begin{aligned}
& -\lim _{\eta \rightarrow 0} \frac{1}{\eta^{2}} \int_{\Sigma_{\eta}} \pi^{1,2} E^{\eta}(x) \cdot \nabla_{\left(y_{1}, y_{2}\right)}^{\perp} \theta_{Y}\left(\frac{x_{1}}{\eta}, \frac{x_{2}}{\eta}\right) \psi(x) d x \\
& =\lim _{\eta \rightarrow 0} \frac{i}{\kappa} \int_{\Sigma_{\eta}} \pi^{1,2}\left(J^{\eta}(x)-\eta E^{\eta}(x)\right) \cdot \nabla_{\left(y_{1}, y_{2}\right)}^{\perp} \theta_{Y}\left(\frac{x_{1}}{\eta}, \frac{x_{2}}{\eta}\right) \psi(x) d x \\
& =\frac{i}{\kappa} \int_{\Omega} \psi(x) \int_{\Sigma} \nabla_{\left(y_{1}, y_{2}\right)}^{\perp} \Phi\left(x, y_{1}, y_{2}\right) \cdot \nabla_{\left(y_{1}, y_{2}\right)}^{\perp} \theta_{Y}\left(y_{1}, y_{2}\right) d y_{1} d y_{2} d x \\
& =\frac{-1}{\omega \varepsilon_{0} \kappa} \sum_{k=0}^{3} \int_{\Omega} \psi(x) D_{k}^{J} h_{k}(x) d x,
\end{aligned}
$$

applying the two scale convergence with concentration for $J^{\eta}$ with the limit density $j_{0}=$ $\nabla_{\left(y_{1}, y_{2}\right)}^{\perp} \Phi\left(x, y_{1}, y_{2}\right)$, and using formula (3.38) in the last line.

It remains to evaluate the right hand side of (1.1), tested with $\frac{1}{\eta} \varphi_{\eta}(x) \psi(x)$. We obtain

$$
\begin{aligned}
& i \omega \mu_{0} \frac{1}{\eta} \int_{\Omega} H^{\eta}(x) \cdot \varphi_{\eta}(x) \psi(x) d x=i \omega \mu_{0} \frac{1}{\eta} \int_{\Sigma_{\eta} \cup \Sigma_{\eta}^{\text {inn }} \cup \Gamma_{\eta}} e_{3} \cdot H^{\eta}(x) \theta_{Y}\left(\frac{x_{1}}{\eta}, \frac{x_{2}}{\eta}\right) \psi(x) d x \\
& \rightarrow i 2 \beta \omega \mu_{0} \int_{\Omega} \psi(x) \int_{\Sigma \cup \Sigma^{\text {inn }}} e_{3} \cdot H_{0}\left(x, y_{1}, y_{2}\right) \theta_{Y}\left(y_{1}, y_{2}\right) d y_{1} d y_{2} d x \\
& =i 2 \beta \omega \mu_{0} \sum_{k=0}^{3} \int_{\Omega} \psi(x) D_{k}^{H} h_{k}(x) d x
\end{aligned}
$$

applying the trace Lemma 2.3 in the second line and the representation (3.37) in the last line. This concludes the proof of Lemma 4.1.

The next lemma provides a characterization that confirms the physical intuition: the average of the electric field across the slit equals the strength of the current in the ring and can hence be expressed by the pre-factor $h_{0}(x)$. In mathematical terms, we have the following result. 
Lemma 4.2 (Electric field in the slit II). Let the two-scale limit of $H^{\eta}$ be characterized with coefficients $h_{k}(x)$ as in (3.37). Then, for an arbitrary localization function $\psi \in$ $C_{c}^{\infty}(\Omega)$, the electric field in the slit has the average

$$
\lim _{\eta \rightarrow 0} \frac{1}{2 \alpha \eta^{2}} \int_{\Gamma_{\eta}} e_{1} \cdot E^{\eta}(x) \psi(x) d x=-\frac{i}{\omega \varepsilon_{0}} \int_{\Omega} \psi(x) h_{0}(x) d x .
$$

Proof. The proof is quite similar to the evaluation of slit averages in Lemma 3.3. The main difference is that we now want to evaluate an integral over all of $\Gamma_{\eta}$, not only an integral over a subset. The principal idea is once more to start from the relation $\nabla_{x} \cdot J^{\eta}=0$ and to choose an appropriate test-function.

In the $y_{1}$-direction we choose a smooth function $\tilde{\vartheta}:[0,1 / 2) \rightarrow \mathbb{R}$ with $\tilde{\vartheta}(0)=1$ and with compact support. The trivial extension to $y_{1}<0$ is denoted by $\vartheta$. We modify this function to a continuous function with large gradients, setting

$$
\vartheta^{\eta}\left(y_{1}\right):= \begin{cases}\left(2 \alpha \eta^{3}\right)^{-1}\left(y_{1}+\alpha \eta^{3}\right) & \text { for }\left|y_{1}\right| \leq \alpha \eta^{3}, \\ \tilde{\vartheta}\left(y_{1}-\alpha \eta^{3}\right) & \text { for } y_{1}>\alpha \eta^{3} \\ 0 & \text { for } y_{1}<-\alpha \eta^{3}\end{cases}
$$

For the $y_{2}$-direction along the slit we choose $\theta_{\delta}: \mathbb{R} \rightarrow \mathbb{R}$ with $\theta_{\delta}\left(y_{2}\right)=1$ for $\left(0, y_{2}\right) \in \Gamma$, such that the support of $\theta_{\delta}$ is confined to a $\delta$-ball around this set. Finally, in the $y_{3^{-}}$ direction, we choose $\phi_{\delta}^{\eta}: \mathbb{R} \rightarrow \mathbb{R}$ with $\phi_{\delta}^{\eta}\left(y_{3}\right)=1$ for $y_{3} \in[-\beta \eta, \beta \eta]$ and $\phi_{\delta}^{\eta}\left(y_{3}\right)=0$ for $\left|y_{3}\right|>(\beta+\delta) \eta$. We now define our oscillating test-function as

$$
\varphi_{\eta}^{\delta}(x):=\vartheta^{\eta}\left(x_{1} / \eta\right) \theta_{\delta}\left(x_{2} / \eta\right) \phi_{\delta}^{\eta}\left(x_{3} / \eta\right) \psi(x) .
$$

The claim of (4.7) is a consequence of

$$
\begin{aligned}
0 & =\int_{\Omega} \nabla_{x} \cdot J^{\eta} \eta \varphi_{\eta}^{\delta}=\int_{\Omega} J^{\eta} \cdot \eta \nabla_{x} \varphi_{\eta}^{\delta}=\int_{\Omega} J^{\eta} \cdot \nabla_{y}\left(\vartheta^{\eta} \theta_{\delta} \phi_{\delta}^{\eta}\right) \psi+o(1) \\
& =\int_{\Gamma_{\eta}} J^{\eta}(x) \cdot e_{1} \frac{1}{2 \alpha \eta^{3}} \psi(x) d x+\int_{\Sigma_{\eta}} J^{\eta}(x) \cdot \nabla_{y}\left(\vartheta \theta_{\delta}\right)\left(x_{1} / \eta-\alpha \eta^{3}, x_{2} / \eta\right) \psi(x) d x+\rho(\delta, \eta) .
\end{aligned}
$$

We will give below the arguments that show that $\lim _{\delta \rightarrow 0} \lim _{\eta \rightarrow 0} \rho(\delta, \eta)=0$. Before we do so, let us draw conclusions from the above relation.

We note that the first integral on the right hand side coincides, because of $J^{\eta}=\eta E^{\eta}$ in the slit, with the expression on the left hand side of (4.7). The second integral converges, by the two-scale convergence with concentration of $J^{\eta}$, and the smoothness of $\partial_{y_{1}} \tilde{\vartheta}$, to the double integral

$$
\begin{aligned}
& \int_{\Sigma_{\eta}} J^{\eta}(x) \cdot \nabla_{y}\left(\vartheta \theta_{\delta}\right)\left(x_{1} / \eta-\alpha \eta^{3}, x_{2} / \eta\right) \psi(x) d x \\
& \quad \rightarrow \int_{\Omega} \int_{\Sigma} j_{0}\left(x, y_{1}, y_{2}\right) \cdot \nabla_{y}\left(\vartheta \theta_{\delta}\right)\left(y_{1}, y_{2}\right) d y_{1} d y_{2} \psi(x) d x \\
& \quad=-\int_{\Omega} \int_{\Gamma} \pi^{1} j_{0}\left(x, 0, y_{2}\right) d y_{2} \psi(x) d x=-\int_{\Omega} \int_{\Gamma} \sum_{k=0}^{3} h_{k}(x) \pi^{1} j^{k}\left(0, y_{2}\right) \theta_{\delta}\left(y_{2}\right) d y_{2} \psi(x) d x
\end{aligned}
$$

We now insert $\pi^{1} j^{k}\left(0, y_{2}\right)=-\partial_{y_{2}} \Phi^{k}\left(0, y_{2}\right)$, the normalization $\left.\Phi^{k}\right|_{\partial \Sigma}=0$ for $k=1,2,3$ and, for $k=0$, the normalization $\left.\left(-i \omega \varepsilon_{0}\right) \Phi^{0}\right|_{\partial_{o} \Sigma}=1$ and $\left.\Phi^{0}\right|_{\partial_{i} \Sigma}=0$, see Proposition 
3.1. The integral $\int_{\Gamma} \partial_{y_{2}} \Phi^{k}\left(0, y_{2}\right)$ therefore vanishes for $k \neq 0$ and it is equal to $i /\left(\omega \varepsilon_{0}\right)$ for $k=0$. This provides the claim of (4.7).

Analysis of error terms. It remains to verify $\lim _{\delta \rightarrow 0} \lim _{\eta \rightarrow 0} \rho(\delta, \eta)=0$. One of the error terms in $\rho(\delta, \eta)$ is

$$
\int_{\Omega \backslash \Sigma_{\eta}}\left|J^{\eta}(x)\right| \partial_{y_{2}} \theta_{\delta}\left(x_{2} / \eta\right) d x \leq C \frac{\eta}{\delta} \int_{\Omega}\left|E^{\eta}(x)\right| \rightarrow 0 .
$$

Another error term is due to an integral over $\partial_{y_{1}} \vartheta$ in the extensions $S_{\eta}^{\delta}$. We denote here by $S_{\eta}^{\delta}$ only the relevant part, which extends in each cell by $\delta$ in the $y_{2}$-direction and by $\delta \eta$ in the $y_{3}$-direction, it is of width $2 \alpha \eta^{3}$ in $y_{1}$-direction. This allows to calculate with the Cauchy-Schwarz inequality

$$
\int_{S_{\eta}^{\delta} \backslash \Gamma_{\eta}} \frac{1}{\eta^{2}}\left|E^{\eta}(x)\right| d x \leq \frac{C}{\eta^{2}}\left\|E^{\eta}\right\|_{L^{2}(\Omega)}\left|\left(S_{\eta}^{\delta} \backslash \Gamma_{\eta}\right)\right|^{1 / 2} \leq \frac{C}{\eta^{2}}\left|\eta^{3} \cdot \delta \cdot \delta \eta\right|^{1 / 2} \leq C \delta .
$$

The integrals which are due to contributions of $\partial_{y_{3}} \phi_{\delta}^{\eta}$ for $\left|y_{3}\right|>\beta \eta$ are treated similarly, they have actually already been analyzed in Lemma 3.3.

With the results of the above lemmas we are now able to establish a linear relation between the components $h_{k}(x), k \in\{1,2,3\}$ and the averaged strength of the electric field in the ring $h_{0}(x)$. A comparison of the expressions of Lemma 4.1 and Lemma 4.2 yields, since the function $\psi$ was arbitrary, the following relation between the factors $h_{k}(x)$.

Corollary 4.1 (Frequency dependent relation between the factors $h_{k}(x)$ and $h_{0}(x)$ ). For almost every $x \in \Omega$, with the constants $D_{k}^{H}, D_{k}^{J}$ of (4.4)-(4.5), using once more $k_{0}^{2}:=\omega^{2} \mu_{0} \varepsilon_{0}$, there holds

$$
\sum_{k=0}^{3}\left(2 \beta k_{0}^{2} D_{k}^{H}-\frac{i}{\kappa} D_{k}^{J}\right) h_{k}(x)=\frac{2 \alpha}{L(\Gamma)} h_{0}(x) .
$$

The averaged strength of the electric field in the ring, $h_{0}(x)$, can therefore be written in terms of $h_{k}(x), k \in\{1,2,3\}$, as

$$
h_{0}(x)=\sum_{k=1}^{3} \lambda_{k} h_{k}(x),
$$

where the three constants $\lambda_{k}$ are defined by

$$
\lambda_{k}:=-\frac{2 \beta \omega^{2} \mu_{0} \varepsilon_{0} D_{k}^{H}-i D_{k}^{J} / \kappa}{2 \beta \omega^{2} \mu_{0} \varepsilon_{0} D_{0}^{H}-i D_{0}^{J} / \kappa-2 \alpha / L(\Gamma)} .
$$

At this point, we have a complete description of the microscopic behavior of the magnetic field. We recall that the magnetic field $H(x)$ is constructed as a weak limit of the solutions $H^{\eta}$, hence the three components $H_{1}(x), \ldots, H_{3}(x)$ of $H(x)$ provide the averaged strength of the magnetic field in the three directions. Since $Y$-averages of the two-scale limit provide the weak limit of a sequence, we conclude that the coefficients $h_{1}(x), \ldots, h_{3}(x)$ must coincide with the averaged magnetic field components $H_{1}(x), \ldots, H_{3}(x)$. The remaining coefficient $h_{0}(x)$ is then determined by (4.11). We have the two-scale limit of the magnetic field characterized as

$$
H_{0}(x, y)=H_{1}(x) H^{1}(y)+H_{2}(x) H^{2}(y)+H_{3}(x) H^{3}(y)+\left(\sum_{k=1}^{3} \lambda_{k} H_{k}(x)\right) H^{0}(y) .
$$




\subsection{Proof of the main theorem}

We use the three cell-problem solutions $E^{l}(y), l=1,2,3$ of (3.9), and the four cell-problem solutions $H^{k}(y), k=0,1,2,3$ of Proposition 3.1 to define effective tensors as follows. The matrix $\mathcal{N} \in \mathbb{C}^{3 \times 3}$ is defined as

$$
\mathcal{N}_{k, l}:=\int_{Y} E^{k}(y) \cdot E^{l}(y) d y
$$

Four circulation vectors $M_{k} \in \mathbb{C}^{3}, k=0,1,2,3$, are defined by

$$
M_{k} \cdot e_{l}:=\int_{\Gamma_{l}} H^{k}(y) \cdot e_{l} d \mathcal{H}^{1}(y) \quad \text { for } l=1,2,3 .
$$

In this definition, $\Gamma_{l}$ is a line that does not intersect $\operatorname{conv}\left(\Sigma_{Y}^{2}\right)$, it connects the faces $\left\{y_{l}=-1 / 2\right\}$ and $\left\{y_{l}=1 / 2\right\}$ and the end-points are identified in the periodic setting. The fact that the curl of $H^{k}$ vanishes outside $\Sigma_{Y}^{2}$ implies that the definition of the circulation vectors $M_{k}$ is independent of the choice of the line $\Gamma_{l}$. From the vectors $M_{k}$ we obtain the effective matrix $\mathcal{M} \in \mathbb{C}^{3 \times 3}$ as

$$
\mathcal{M}_{k, l}:=\left(M_{k}+\lambda_{k} M_{0}\right) \cdot e_{l}
$$

for $k, l=1,2,3$.

The effective macroscopic problem. In order to prove Theorem 1, it remains to verify the effective equation (1.9), which is the averaged version of (1.2), i.e.

$$
\operatorname{curl} H^{\eta}=-i \omega \varepsilon_{\eta} \varepsilon_{0} E^{\eta}
$$

All we have to do is to construct an appropriate oscillating test-function for this relation. Within the cell $Y$ we use a cut-off function $\vartheta \in C_{\text {per }}^{\infty}(Y)$ with the property that $\vartheta \equiv 1$ in a neighborhood of the cell-boundary $\partial Y$, and with $\vartheta \equiv 0$ in a neighborhood of $\operatorname{conv}\left(\Sigma_{Y}^{2}\right)$. With the coordinate function $y_{l}$, we then use

$$
p^{l}(y):=\nabla_{y}\left[y_{l} \vartheta(y)\right], \quad \operatorname{supp}\left(p^{l}\right) \cap \operatorname{conv}\left(\Sigma_{Y}^{2}\right)=\emptyset, \operatorname{curl}_{y} p^{l} \equiv 0, \int_{Y} p^{l}=e_{l} .
$$

For the localization in $x$ we choose $\psi \in C_{c}^{\infty}(Q)$. We finally choose as an oscillating test-function in (4.17) the function $\varphi_{\eta}(x):=\psi(x) p^{l}(x / \eta)$.

We first calculate the result, when the right hand side of (4.17) is tested with $\varphi_{\eta}$. Since $p^{l}$ vanishes on $\Sigma_{\eta}$, the relative permittivity $\varepsilon_{\eta}$ is identical to 1 on the support of the test-function. Using the characterization of the two-scale limit $E_{0}(x, y)$ from (3.8), we find

$$
\begin{aligned}
& \int_{Q}\left(-i \omega \varepsilon_{0} \varepsilon_{\eta} E^{\eta}\right)(x) \cdot \varphi_{\eta}(x) d x=-i \omega \varepsilon_{0} \int_{Q} E^{\eta}(x) \cdot p^{l}(x / \eta) \psi(x) d x \\
& \quad \rightarrow-i \omega \varepsilon_{0} \sum_{k=1}^{3} \int_{\Omega} \psi(x) E_{k}(x) \int_{Y} E^{k}(y) \cdot p^{l}(y) d y d x-i \omega \varepsilon_{0} \sum_{k=1}^{3} \int_{Q \backslash \Omega} \psi(x) E_{l}(x) d x \\
& \quad=-i \omega \varepsilon_{0} \int_{Q} \psi(x) \sum_{k=1}^{3} \hat{N}_{k, l} E_{k}(x) d x
\end{aligned}
$$


where $\mathcal{N}$ was defined in (4.14) and $\hat{N}(x)$ is constructed from $\mathcal{N}$ as in (1.10). In the last equation we exploited the fact that scalar product of the $E$-field with $p^{l}$ is identical to scalar product with $E^{l}$. We use the representation of the cell solution $E^{k}(y):=e_{k}+\nabla \phi^{k}(y)$ from (3.9) to calculate with integration by parts

$$
\begin{gathered}
\int_{Y} E^{k}(y) \cdot\left(E^{l}(y)-p^{l}(y)\right) d y=\int_{Y} E^{k}(y) \cdot \nabla_{y}\left(y_{l}+\phi^{l}(y)-\left[y_{l} \vartheta(y)\right]\right) d y \\
=-\int_{\Sigma_{Y}^{2}}\left[E^{k}(y) \cdot e_{3}\right]_{\Sigma}\left(y_{l}+\phi^{l}(y)\right) d \mathcal{H}^{2}(y)=0,
\end{gathered}
$$

using in the last equality that $y_{l}+\phi^{l}(y)$ vanishes on $\Sigma_{Y}^{2}$. We now evaluate the left hand side of (4.17), tested with $\varphi_{\eta}$. We integrate by parts, use that the fine-scale curl of the test-function vanishes, $\operatorname{curl}_{y} p^{l}(y)=0$, and obtain from the characterization of the two-scale limit $H_{0}(x, y)=\sum_{k=1}^{3} H_{k}(x) H^{k}(y)+\sum_{k=1}^{3} \lambda_{k} H_{k}(x) H^{0}(y)$ in (4.13)

$$
\begin{gathered}
\int_{Q} \operatorname{curl} H^{\eta} \cdot \varphi_{\eta}=-\int_{Q} H_{\eta}(x) \cdot p^{l}(x / \eta) \wedge \nabla \psi(x) d x \rightarrow-\int_{Q \backslash \Omega} H(x) \wedge e_{l} \cdot \nabla \psi(x) d x \\
-\int_{\Omega} \sum_{k=1}^{3} H_{k}(x) \int_{Y}\left(H^{k}(y)+\lambda_{k} H^{0}(y)\right) \wedge p^{l}(y) \cdot \nabla \psi(x) d y d x .
\end{gathered}
$$

To evaluate the $Y$-integral, we calculate for $k=0,1,2,3$, exploiting $\operatorname{curl}_{y} H^{k}=0$ on $Y \backslash \bar{\Sigma}_{Y}^{2}$

$$
\begin{gathered}
\int_{Y} H^{k}(y) \wedge p^{l}(y) d y=\int_{Y} H^{k}(y) \wedge \nabla_{y}\left[y_{l} \vartheta(y)\right] d y=-\int_{Y} \operatorname{curl}_{y}\left(H^{k}(y) y_{l} \vartheta(y)\right) d y \\
=-\int_{\partial Y} \nu \wedge H^{k}(y) y_{l} d \mathcal{H}^{2}(y)=-\int_{\left\{y_{l}=1 / 2\right\}} e_{l} \wedge H^{k}(y) d \mathcal{H}^{2}(y)=-e_{l} \wedge M_{k}
\end{gathered}
$$

with the circulation vector $M_{k}$ of (4.15).

We can now compare the two expressions that were obtained from testing (4.17). Recalling that we extended $\mathcal{N}$ and $\mathcal{M}$ by unit matrices outside $\Omega$ to define $\hat{N}$ and $\hat{M}$, see (1.10), there holds

$$
\begin{aligned}
-i \omega & \varepsilon_{0} \int_{Q} \psi(x) \hat{N}(x) \cdot E(x) d x \\
= & \int_{Q \backslash \Omega} e_{l} \wedge H(x) \cdot \nabla \psi(x) d x+\int_{\Omega} e_{l} \wedge\left(\sum_{k=1}^{3} H_{k}(x)\left(M_{k}+\lambda_{k} M_{0}\right)\right) \cdot \nabla \psi(x) d x \\
= & \int_{Q} e_{l} \cdot \operatorname{curl}_{x}(\hat{M}(x) H(x)) \psi(x) d x .
\end{aligned}
$$

The localization function $\psi$ was arbitrary and the index $l \in\{1,2,3\}$ was arbitrary, hence we obtain, in the distributional sense on $Q$,

$$
-i \omega \varepsilon_{0} \hat{N}(x) \cdot E(x)=\operatorname{curl}_{x}(\hat{M}(x) \cdot H(x)) .
$$

This was the claim in the effective equation (1.9) and we have thus shown Theorem 1. 


\subsection{Sign-properties of the effective coefficients}

In order to show that the proposed design can generate indeed a negative permeability, we conclude this study with an investigation of sign properties of the effective matrix $\mathcal{M}$ of (4.16) in a limiting case. We consider the entry $\mathcal{M}_{3,3}$ and claim that, for appropriate parameters $\alpha, \beta$, and $\kappa$, the real part $\Re\left(\mathcal{M}_{3,3}\right)$ can have both signs, depening on the frequency $\omega>0$.

We will not perform a rigorous proof for the above claim. Instead, we will show the following. We consider the cell problem which is obtained formally by choosing extreme parameters $\alpha$ and $\kappa$. We study solutions to this simplified cell problem and show that, evaluating the circulation vectors $M_{k}$ and the coefficients $D_{k}^{H}$ for those solutions, the corresponding entry $\mathcal{M}_{3,3}$ has a real part of arbitrary sign.

Our original interest is the cell-problem (3.33)-(3.36). We send, formally, $\kappa \rightarrow \infty$ and $\alpha \rightarrow 0$. Then (3.36) reduces to the relation $\operatorname{tr}_{\Sigma}^{3} H_{0}=0$, and the simplified system reads

$$
\begin{aligned}
\operatorname{div}_{y} H_{0}=0 & \text { in } Y \\
\operatorname{curl}_{y} H_{0}=0 & \text { in } Y \backslash \bar{\Sigma}_{Y}^{2} \\
e_{3} \cdot H_{0}=0 & \text { on } \Sigma_{Y}^{2} .
\end{aligned}
$$

The normalization of the four special solutions $H^{0}(y), \ldots, H^{3}(y)$ from (3.39)-(3.40) is as follows. The $Y$-averages of $H^{k}$ are $e_{k}$ for $k \geq 1$ and vanish for $H^{0}$. For the other normalization, we study a closed curve $\Gamma_{r}$, winding once through the ring, with tangential vector $\tau_{r}$ such that $\tau_{r}=e_{3}$ in the point $\Gamma_{r} \cap \Sigma^{\text {inn }}$. Then there holds, for $k \geq 0$,

$$
\int_{Y} H^{k} \cdot e_{l}=\delta_{k, l} \quad l=1,2,3, \quad \int_{\Gamma_{r}} H^{k}(y) \cdot \tau_{r}(y) d \mathcal{H}^{1}(y)=\delta_{k, 0} .
$$

The next lemma investigates sign properties for the solutions of the above reduced cellproblem.

Lemma 4.3. Let $H^{0}, \ldots, H^{3}$ be the four solutions of system (4.19)-(4.21), normalized by (4.22). We consider the corresponding circulation vectors $M_{k}$ of (4.15) and the coefficients $D_{k}^{H}, D_{k}^{J}$ as in (4.4)-(4.5). Then there holds

$$
M_{k}, D_{k}^{H}, D_{k}^{J} \text { are real for all } k=0,1,2,3, \text { with }-e_{3} \cdot M_{0}, e_{3} \cdot M_{3}, D_{0}^{H}, D_{3}^{H}>0 \text {. }
$$

Once the lemma is shown, we can conclude the following. The definition of $\lambda_{k}$ in (4.12) implies that, for a large conductivity constant $\kappa>0$ and appropriate choices of $\alpha$ and $\beta$, the factors $\lambda_{k}$ can have large absolute value and the real part can have both signs. By the definition of $\mathcal{M}$ in (4.16), the number $\Re\left(\mathcal{M}_{3,3}\right)$ can then have both signs.

Proof. The fact that the solutions are real is due to the real character of equations (4.19)(4.22). The solutions $H^{k}$ can be constructed almost explicitely as gradients. We will use periodic functions $\Psi: Y \rightarrow \mathbb{R}$ solving

$$
\begin{array}{rlrl}
\Delta \Psi & =0 & & \text { on } Y \backslash Y^{2}, \\
e_{3} \cdot \nabla \Psi=0 & & \text { on } \Sigma_{ \pm} .
\end{array}
$$

with periodic boundary conditions on $\partial Y$. We then set $H=\nabla \Psi$. With this construction, $H$ solves (4.19) outside $Y^{2}$ and (4.20)-(4.21). In order to obtain a solution $\tilde{H}^{0}=\nabla \Psi^{0}$, we use $\Psi=\Psi^{0}$ that satisfies the further boundary conditions, using $\Sigma_{c}=\Sigma \cup \Sigma^{\mathrm{inn}}$,

$$
\begin{array}{ll}
\Psi^{0}=\mp 1 & \text { on }\left(\Sigma^{\text {inn }}\right)_{ \pm}, \\
\Psi^{0}= \pm \mu & \text { on }\left(Y^{2} \backslash \Sigma_{c}\right)_{ \pm},
\end{array}
$$


for some $\mu>0$. We note that $\Psi^{0}$ is anti-symmetric with respect to $y_{3}=0$, there holds $\Psi^{0}\left(y_{1}, y_{2}, y_{3}\right)=-\Psi^{0}\left(y_{1}, y_{2},-y_{3}\right)$. This implies that $\tilde{H}^{0} \cdot e_{3}$ is without jump across $Y^{2}$, hence $\tilde{H}^{0}$ has vanishing divergence on all of $Y$. Regarding the normalization, we can achieve, using the trace from $y_{3}>0$,

$$
\int_{Y} \tilde{H}^{0}(y) \cdot e_{3} d y=-2 \int_{Y^{2}} \Psi^{0}\left(y_{1}, y_{2}\right) d y_{1} d y_{2}=0
$$

with an appropriate choice of $\mu>0$. Since $\tilde{H}^{0}$ is non-trivial and the other components of the average of $\tilde{H}^{0}$ also vanish, we obtained, up to a multiplicative factor, the desired function $H^{0}$. Concerning the multiplicative factor, we calculate

$$
\int_{\Gamma_{r}} \tilde{H}^{0}(y) \cdot \tau_{r}(y) d \mathcal{H}^{1}(y)=2 \mu+2
$$

hence we obtain the cell solution as $H^{0}:=(2 \mu+2)^{-1} \tilde{H}^{0}$. The constant $D_{0}^{H}$ from (4.4) is

$$
D_{0}^{H}=\int_{\Sigma_{c}} e_{3} \cdot H^{0}\left(y_{1}, y_{2}, 0\right) \theta_{Y}\left(y_{1}, y_{2}\right) d y_{1} d y_{2}=(2 \mu+2)^{-1} \int_{\Sigma^{\mathrm{inn}}} \partial_{3} \Psi^{0} \theta_{Y}>0
$$

and the circulation vector $M_{0} \in \mathbb{C}^{3}$ of $(4.15)$ is

$$
M_{0} \cdot e_{3}=\int_{\Gamma_{3}} H^{0}(y) \cdot e_{3} d \mathcal{H}^{1}(y)=-2 \mu<0
$$

Similarly, we obtain $H^{3}$. We solve (4.24)-(4.25), now with $\Psi=\Psi^{3}$ satisfying

$$
\Psi^{3}=\mp 1 \quad \text { on }\left(Y^{2} \backslash \Sigma\right)_{ \pm} .
$$

Since $\Psi^{3}$ has equal jumps in $\Sigma^{\text {inn }}$ and in $Y^{2} \backslash \Sigma_{c}$, the curve integrals over $\tilde{H}^{3}=\nabla \Psi^{3}$ vanish. Since gradients of $\Psi^{3}$ point in positive $y_{3}$-directions, we obtain $H^{3}=\nu \tilde{H}^{3}$ with a positive factor $\nu>0$. The corresponding constants are

$$
D_{3}^{H}=\int_{\Sigma_{c}} e_{3} \cdot H^{3}\left(y_{1}, y_{2}, 0\right) \theta_{Y}\left(y_{1}, y_{2}\right) d y_{1} d y_{2}=\nu \int_{\Sigma_{\text {inn }}} \partial_{3} \Psi^{3} \theta_{Y}>0
$$

and the circulation vector $M_{3} \in \mathbb{C}^{3}$ of (4.15) has

$$
M_{3} \cdot e_{3}=\int_{\Gamma_{3}} H^{3}(y) \cdot e_{3} d \mathcal{H}^{1}(y)>0 .
$$

This shows the lemma.

\section{References}

[1] G. Allaire. Homogenization and two-scale convergence. SIAM J. Math. Anal., 23(6):1482-1518, 1992.

[2] M. Bellieud and I. Gruais. Homogenization of an elastic material reinforced by very stiff or heavy fibers. Non-local effects. Memory effects. J. Math. Pures Appl. (9), 84(1):55-96, 2005. 
[3] G. Bouchitté, C. Bourel, and D. Felbacq. Homogenization of the 3D Maxwell system near resonances and artificial magnetism. C. R. Math. Acad. Sci. Paris, 347(910):571-576, 2009.

[4] G. Bouchitté and D. Felbacq. Low frequency scattering by a set of parallel metallic rods. In Mathematical and numerical aspects of wave propagation (Santiago de Compostela, 2000), pages 226-230. SIAM, Philadelphia, PA, 2000.

[5] G. Bouchitté and D. Felbacq. Homogenization near resonances and artificial magnetism from dielectrics. C. R. Math. Acad. Sci. Paris, 339(5):377-382, 2004.

[6] G. Bouchitté and D. Felbacq. Homogenization of a wire photonic crystal: the case of small volume fraction. SIAM J. Appl. Math., 66(6):2061-2084, 2006.

[7] G. Bouchitté and B. Schweizer. Cloaking of small objects by anomalous localized resonance. Quart. J. Mech. Appl. Math., 63(4):437-463, 2010.

[8] G. Bouchitté and B. Schweizer. Homogenization of Maxwell's equations with split rings. SIAM Multiscale Modeling and Simulation, 8(3):717-750, 2010.

[9] K. D. Cherednichenko, V. P. Smyshlyaev, and V. V. Zhikov. Non-local homogenized limits for composite media with highly anisotropic periodic fibres. Proc. Roy. Soc. Edinburgh Sect. A, 136(1):87-114, 2006.

[10] D. Felbacq and G. Bouchitté. Homogenization of a set of parallel fibres. Waves Random Media, 7(2):245-256, 1997.

[11] S. Guenneau, F. Zolla, and A. Nicolet. Homogenization of 3D finite photonic crystals with heterogeneous permittivity and permeability. Waves Random Complex Media, 17(4):653-697, 2007.

[12] R. Kohn, H. Shen, M. Vogelius, and M. Weinstein. Cloaking via change of variables in electric impedance tomography. Inverse Problems, 24(1):015016, 21, 2008.

[13] R. Kohn and S. Shipman. Magnetism and homogenization of micro-resonators. Multiscale Modeling ES Simulation, 7(1):62-92, 2007.

[14] R. V. Kohn, D. Onofrei, M. S. Vogelius, and M. I. Weinstein. Cloaking via change of variables for the Helmholtz equation. Comm. Pure Appl. Math., 63(8):973-1016, 2010 .

[15] A. Lamacz. Dispersive effective models for waves in heterogeneous media. Math. Models Methods Appl. Sci., 21(9):1871-1899, 2011.

[16] M. Mihailovici and B. Schweizer. Effective model for the cathode catalyst layer in fuel cells. Asymptot. Anal., 57(1-2):105-123, 2008.

[17] G. Milton and N.-A. Nicorovici. On the cloaking effects associated with anomalous localized resonance. Proc. R. Soc. Lond. Ser. A Math. Phys. Eng. Sci., 462(2074):30273059, 2006.

[18] G. Milton and P. Seppecher. Realizable response matrices of multi-terminal electrical, acoustic and elastodynamic networks at a given frequency. Proc. R. Soc. Lond. Ser. A Math. Phys. Eng. Sci., 464(2092):967-986, 2008. 
[19] A. B. Movchan and S. Guenneau. Split-ring resonators and localized modes. Phys. Rev. B, 70(12):125116, Sep 2004.

[20] G. Nguetseng. A general convergence result for a functional related to the theory of homogenization. SIAM J. Math. Anal., 20(3):608-623, 1989.

[21] S. O'Brien and J. Pendry. Magnetic activity at infrared frequencies in structured metallic photonic crystals. J. Phys. Condens. Mat., 14:6383-6394, 2002.

[22] J. Pendry, A. Holden, D. Robbins, and W. Stewart. Magnetism from conductors and enhanced nonlinear phenomena. IEEE Trans. Microwave Theory Tech., 47(2075), 1999 .

[23] B. Schweizer. Homogenization of degenerate two-phase flow equations with oil trapping. SIAM J. Math. Anal., 39(6):1740-1763, 2008.

[24] D. Smith, J. Pendry, and M. Wiltshire. Metamaterials and negative refractive index. Science, 305:788-792, 2004.

[25] V. Veselago. The electrodynamics of substances with simultaneously negative values of $\varepsilon$ and $\mu$. Soviet Physics Uspekhi, 10:509-514, 1968. 


\section{Preprints ab 2010/04}

2012-06

2012-05

2012-04

2012-03

2012-02

2012-01

2011-14

$2011-13$

$2011-12$

2011-11

2011-10

2011-09

2011-08

2011-07

2011-06

2011-05

2011-04

Agnes Lamacz and Ben Schweizer

Effective Maxwell equations in a geometry with flat rings of arbitrary shape

Frank Klinker and Günter Skoruppa

Ein optimiertes Glättungsverfahren motiviert durch eine technische Fragestellung

Patrick Henning, Mario Ohlberger, and Ben Schweizer

Homogenization of the degenerate two-phase flow equations

Andreas Rätz

A new diffuse-interface model for step flow in epitaxial growth

Andreas Rätz and Ben Schweizer

Hysteresis models and gravity fingering in porous media

Wilfried Hazod

Intrinsic topologies on $\mathrm{H}$-contraction groups with applications to semistability

Guy Bouchitté and Ben Schweizer

Plasmonic waves allow perfect transmission through sub-wavelength metallic gratings

Waldemar Grundmann

Moment functions and Central Limit Theorem for Jacobi hypergroups on $[0, \infty[$

J. Koch, A. Rätz, and B. Schweizer

Two-phase flow equations with a dynamic capillary pressure

Michael Voit

Central limit theorems for hyperbolic spaces and Jacobi processes on $[0, \infty[$

Ben Schweizer

The Richards equation with hysteresis and degenerate capillary pressure

Andreas Rätz and Matthias Röger

Turing instabilities in a mathematical model for signaling networks

Matthias Röger and Reiner Schätzle

Control of the isoperimetric deficit by the Willmore deficit

Frank Klinker

Generalized duality for k-forms

Sebastian Aland, Andreas Rätz, Matthias Röger, and Axel Voigt

Buckling instability of viral capsides - a continuum approach

Wilfried Hazod

The concentration function problem for locally compact groups revisited: Non-dissipating space-time random walks, $\tau$-decomposable laws and their continuous time analogues

Wilfried Hazod, Katrin Kosfeld

Multiple decomposability of probabilities on contractible locally compact groups 


\section{(2011-01}

2010-16

$2010-15$

2010-14

$2010-13$

$2010-12$

$2010-11$

$2010-10$

2010-09

2010-08

2010-07

2010-06

2010-05

2010-04

\section{Ben Schweizer and Marco Veneroni}

The needle problem approach to non-periodic homogenization

\section{Sebastian Engelke and Jeannette H.C. Woerner}

A unifying approach to fractional Lévy processes

Alexander Schnurr and Jeannette H.C. Woerner

Well-balanced Lévy Driven Ornstein-Uhlenbeck Processes

\section{Lorenz J. Schwachhöfer}

On the Solvability of the Transvection group of Extrinsic

Symplectic Symmetric Spaces

\section{Marco Veneroni}

Stochastic homogenization of subdifferential inclusions via scale integration

Agnes Lamacz, Andreas Rätz, and Ben Schweizer

A well-posed hysteresis model for flows in porous media and applications to fingering effects

\section{Luca Lussardi and Annibale Magni}

$\Gamma$-limits of convolution functionals

\section{Patrick W. Dondl, Luca Mugnai, and Matthias Röger \\ Confined elastic curves}

Matthias Röger and Hendrik Weber

Tightness for a stochastic Allen-Cahn equation

\section{Michael Voit}

Multidimensional Heisenberg convolutions and product formulas

for multivariate Laguerre polynomials

Ben Schweizer

Instability of gravity wetting fronts for Richards equations with hysteresis

\section{Lorenz J. Schwachhöfer}

Holonomy Groups and Algebras

Agnes Lamacz
Dispersive effective models for waves in heterogeneous media

\section{Ben Schweizer and Marco Veneroni}

Periodic homogenization of Prandtl-Reuss plasticity equations in arbitrary dimension 